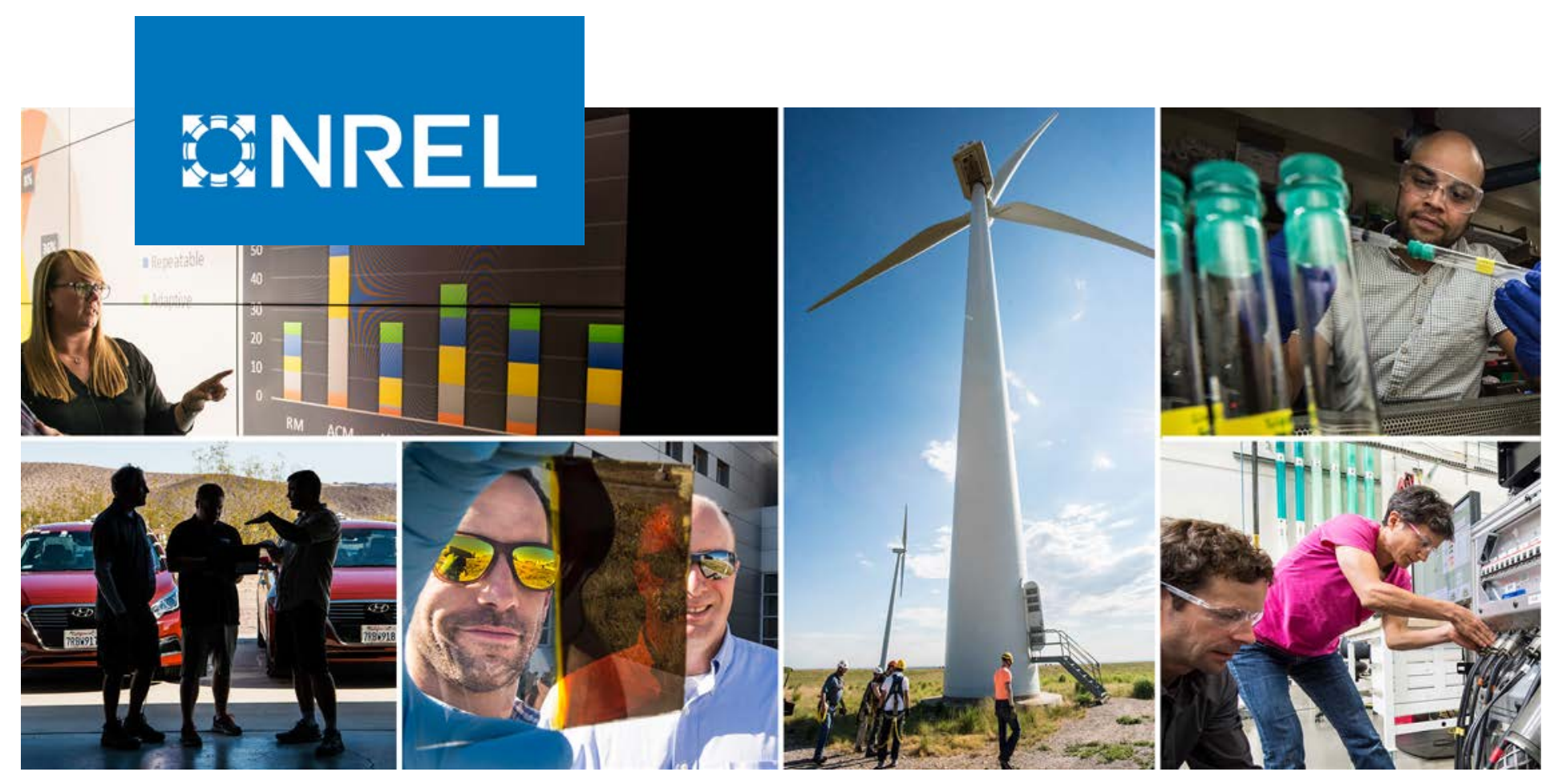

\title{
Comparing Capacity Credit Calculations for Wind: A Case Study in Texas
}

Jennie Jorgenson, Sarah Awara, Gord Stephen, and Trieu Mai

National Renewable Energy Laboratory

NREL is a national laboratory of the U.S. Department of Energy Office of Energy Efficiency \& Renewable Energy

Operated by the Alliance for Sustainable Energy, LLC

This report is available at no cost from the National Renewable Energy Laboratory (NREL) at www.nrel.gov/publications.
Technical Report

NREL/TP-5C00-80486

September 2021 


\title{
GNREL
}

\section{Comparing Capacity Credit}

Calculations for Wind: A Case Study in Texas

\author{
Jennie Jorgenson, Sarah Awara, Gord Stephen, \\ and Trieu Mai
}

National Renewable Energy Laboratory

\section{Suggested Citation)}

Jorgenson, Jennie, Sarah Awara, Gord Stephen, and Trieu Mai. 2021. Comparing Capacity Credit Calculations for Wind: A Case Study in Texas. Golden, CO: National Renewable Energy Laboratory. NREL/TP-5C00-80486.

https://www.nrel.gov/docs/fy21osti/80486.pdf.

NREL is a national laboratory of the U.S. Department of Energy Office of Energy Efficiency \& Renewable Energy Operated by the Alliance for Sustainable Energy, LLC

This report is available at no cost from the National Renewable Energy Laboratory (NREL) at www.nrel.gov/publications.

Contract No. DE-AC36-08GO28308
Technical Report

NREL/TP-5C00-80486

September 2021

National Renewable Energy Laboratory 15013 Denver West Parkway Golden, CO 80401

303-275-3000 • www.nrel.gov 


\section{NOTICE}

This work was authored by the National Renewable Energy Laboratory, operated by Alliance for Sustainable Energy, LLC, for the U.S. Department of Energy (DOE) under Contract No. DE-AC36-08GO28308. Funding provided by the U.S. Department of Energy Office of Energy Efficiency and Renewable Energy Wind Energy Technologies Office. The views expressed herein do not necessarily represent the views of the DOE or the U.S. Government.

This report is available at no cost from the National Renewable Energy Laboratory (NREL) at www.nrel.gov/publications.

U.S. Department of Energy (DOE) reports produced after 1991 and a growing number of pre-1991 documents are available free via www.OSTI.gov.

Cover Photos by Dennis Schroeder: (clockwise, left to right) NREL 51934, NREL 45897, NREL 42160, NREL 45891, NREL 48097, NREL 46526.

NREL prints on paper that contains recycled content. 


\section{Acknowledgments}

We thank Philipp Beiter, Billy Roberts, Sinnott Murphy, Patrick Gilman, Bethany Frew, Eric Lantz, Luke Lavin, Katie Wensuc, Gage Reber, Brian Smith, and Jessica Lau for helpful input during the analysis and publication process. The research was performed using computational resources sponsored by the Department of Energy's Office of Energy Efficiency and Renewable Energy and located at the National Renewable Energy Laboratory. This work was authored by the National Renewable Energy Laboratory, operated by Alliance for Sustainable Energy, LLC, for the U.S. Department of Energy (DOE) under Contract No. DE-AC36-08GO28308. Funding provided by the U.S. Department of Energy Office of Energy Efficiency and Renewable Energy Wind Energy Technologies Office. The views expressed in the article do not necessarily represent the views of the DOE or the U.S. Government. The publisher, by accepting the article for publication, acknowledges that the U.S. Government retains a non-exclusive, paid-up, irrevocable, worldwide license to publish or reproduce the published form of this work, or allow others to do so, for U.S. Government purposes. 


\section{Acronyms}

CC: Capacity credit

CF: Capacity factor

ECC: Equivalent conventional capacity

EFC: Equivalent firm capacity

ELCC: Effective load-carrying capability

EUE: Expected unserved energy

ERCOT: Electric Reliability Council of Texas

FOR: Forced outage rate

LOLE: Loss-of-load expectation

LOLP: Loss-of-load probability

NARIS: North American Renewable Integration Study

NEUE: Normalized expected unserved energy

NREL: National Renewable Energy Laboratory

PRAS: Probabilistic Resource Adequacy Suite

PV: Photovoltaics

ReEDS: Regional Energy Deployment System

WIND Toolkit: Wind Integration National Dataset Toolkit 


\section{Executive Summary}

The degree to which wind power can contribute to resource adequacy requirements, also known as its capacity credit (CC), varies with wind resource and correlation to net load. CC, usually expressed as a percentage relative to a resource's nameplate capacity, is an important metric widely used for resource planning and resource adequacy assessments. Furthermore, CC describes one potential component of the value of wind and thus has implications for future deployment. However, there are multiple methods for estimating CC, depending on specific needs, access to data, and computational resources. For example, probabilistic resource adequacy tools are useful for calculating marginal CCs, but they can require sophisticated methods and data sets. As a result, simpler approximation methods are widely used in capacity expansion models and for energy system planning. To evaluate the importance of method choices, we use a probabilistic resource adequacy tool and multiple approximation methods to systematically assess the CC of wind for near-term deployment in a Texas Interconnection test system. We compare CCs across these methods and discuss how the difference in approaches and assumptions can impact the resulting CCs.

Initially using NREL's Probabilistic Resource Adequacy Suite (PRAS) tool, we observe a relatively low marginal wind $\mathrm{CC}$ in the Texas Interconnection test system that is influenced by substantial existing wind deployment as well as wind patterns that are not well correlated with periods of high net load, usually occurring during the summer when wind output is lower. To weigh the trade-offs associated with the simplifications, we compare our PRAS results to the approximation-based calculations, which involve finding the average capacity factor of a marginal resource during times of elevated system risk but also methodological choices and parameters for both methods. We find that approximation methods can capture the important relationships between wind availability and system needs; however, the chosen approximation method can impact the resulting CCs. An important consideration is transmission - whether or not wind will be able to address reliability needs in neighboring regions. In this case study, we find that several approximation methods do come close to the $\mathrm{CC}$ calculated by more robust probabilistic methods (PRAS), however can vary substantially. PRAS results are less sensitive to modeling criteria in this system. Finally, choosing the correct criteria for approximating the CC can be difficult because the best approximation method can vary on a case-by-case basis and the best method for this Texas case study may not be universal. 


\section{Table of contents}

Acknowledgments $\ldots \ldots \ldots \ldots \ldots \ldots \ldots \ldots \ldots \ldots \ldots \ldots \ldots \ldots \ldots \ldots$

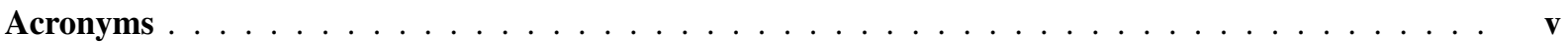

Executive Summary $\ldots \ldots \ldots \ldots \ldots \ldots \ldots \ldots \ldots \ldots \ldots \ldots \ldots$

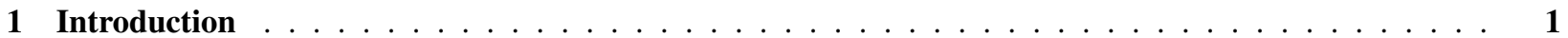

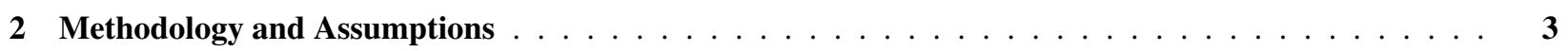

2.1 Texas Interconnection Test System . . . . . . . . . . . . . . . . . . . . . 3

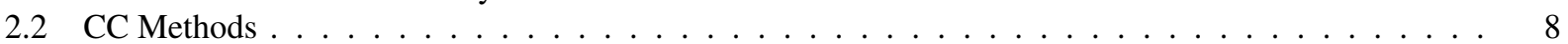

3 Sensitivities on PRAS CC $\ldots \ldots \ldots \ldots \ldots \ldots \ldots$

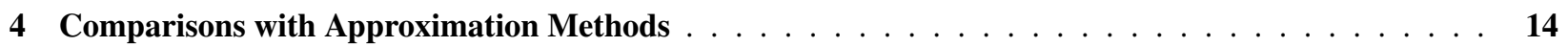

4.1 CC Computed with EUE-Weighted CF . . . . . . . . . . . . . . . . . . . . . . . . 14

4.2 CC Estimated By Measures of Load . . . . . . . . . . . . . . . . . . . . . . 15

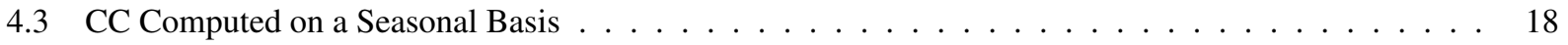

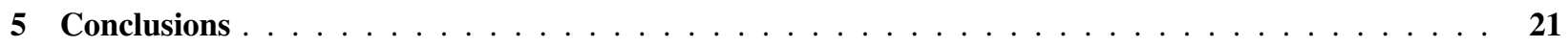

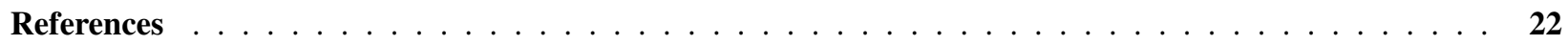

\section{List of Figures}

Figure 1. Map of the modeled footprint, including the seven modeled zones with numbers corresponding to

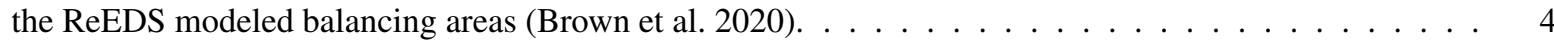

Figure 2. Map of the 7-year average CF calculated from WIND Toolkit data for 2007-2013. . . . . . . . 6

Figure 3. Average diurnal load and net load profiles by month for the Texas Interconnection test system, with ribbons indicating the observed percentiles over the 7 years of data (2007-2013). This reflects our Base Case generator fleet, with $24 \%$ generation contribution from wind and $12 \%$ from solar. . . . . . . .

Figure 4. Average diurnal wind CFs from the 7 years of WIND Toolkit data for all grid cells, with ribbons

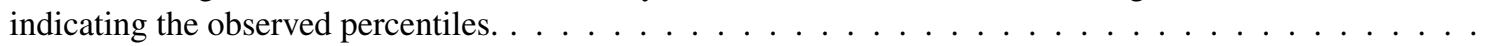

Figure 5. Average diurnal system-wide EUE calculated by PRAS for the 7 years studied here by month,

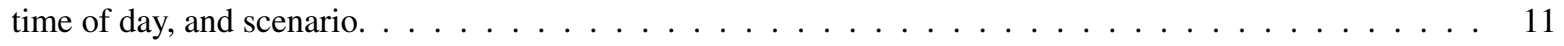

Figure 6. Base Case PRAS-computed marginal $\mathrm{CC}$ for each grid cell in the footprint. . . . . . . . . . . 12

Figure 7. The difference between the Base Case PRAS-computed marginal CC for each grid cell compared to three different variations on the starting PRAS case. A positive indicates the new starting point increased the marginal CC, whereas a negative value represents a reduced $\mathrm{CC}$. 
Figure 8. Comparison of zone EUE- and system EUE-weighted CF to the Base Case PRAS-computed CCs.

Figure 9. The difference between the CC approximation calculated by the CF weighted by either the Texas system-wide EUE or zone EUE and the Base Case PRAS CC. . . . . . . . . . . . . . . . . 15

Figure 10. Comparison of marginal CC approximated by average CF during top 10 hours per year to PRAScomputed CCs. . . . . . . . . . . . . . . . . . . . . . . . . . 17

Figure 11. Comparison of CC approximated by average CF during top hours to PRAS-computed CCs. . . . . 18

Figure 12. Comparison of CC approximated by weighting of CF by hourly Texas system-wide EUE-weighted during each of four seasons. . . . . . . . . . . . . . . . . . . . . . . . 19

Figure 13. Comparison of CC approximated by average CF during the top 10 hours during each of four sea-

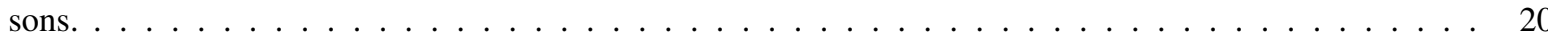

\section{List of Tables}

Table 1. Assumptions for the modeled Base Case generating capacity by type, along with system demand. . 4

Table 2. Turbine specifications used to generate the CF profiles for the incremental wind capacity. . . . . . 5

Table 3. Sensitivities of the starting point for PRAS-calculated CC and on CF approximation methods ex-

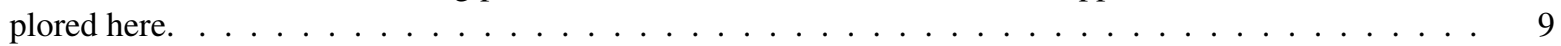




\section{Introduction}

Wind energy capacity in the United States has seen a dramatic increase in recent years, totalling $118 \mathrm{GW}$ through the end of 2020 (U.S. Energy Information Administration 2021). Declining costs and renewable energy goals will likely continue to drive this trend (Stehly, Beiter, and Duffy 2020, Barbose 2021). Because wind generation is variable and weather dependent, evaluating its contribution to power system reliability and adequacy can be complicated. The metric of capacity credit (CC) is often used to facilitate comparisons between the firm capacity offerings of various generation types, including wind. CC quantifies the percentage capacity contribution of a specific resource to overall system adequacy relative to its nameplate capacity (Garrido 2019, Ibanez and Milligan 2014).

Two main methods are used to estimate $\mathrm{CC}^{1}$ : reliability-based and approximation-based methods (Sioshansi, Madaeni, and Denholm 2013). Reliability-based methods typically use resource adequacy models to calculate metrics such as the loss-of-load probability (LOLP) or expected unserved energy (EUE) given inputs such as hourly loads, generator information, and often transmission network data (Corporation 2018, Jorgenson et al. 2021, Mills and Rodriguez 2020). These methods can calculate the CC of an incremental resource by computing the reliability metric before and after the addition of the new resource, with any change in the metric attributed to the incremental resource. Simpler approximation-based methods usually calculate the CC of a unit based on the capacity factor (CF), which is the percentage output of a plant relative to its nameplate capacity, during a predetermined period. This period generally covers the hours where the system is expected to be under stress, such as hours of peak load or peak net load ${ }^{2}$. These methods use an estimate for when capacity is expected to be the most constrained, but they might miss certain factors such as the impact of incremental resources on shifting the highest risk periods and intertemporal constraints on energy-limited resources (Milligan 2000, Milligan 1997). Although both approximationand reliability-based methods might not include transmission constraints, they can usually be considered when using simulation-based Monte Carlo methods ${ }^{3}$. For all these reasons, reliability-based resource adequacy methods are preferred whenever data and computational capabilities are available (Milligan 1997, Keane et al. 2010, Soder and Amelin 2008). Approximation-based methods to calculate CC are still widely used and can provide useful insight, particularly for future system planning with capacity expansion models (Milligan and Porter 2008, Dent, Keane, and Bialek 2010).

Previous work on a case study in the western United States finds that CF approximation methods averaging the top 100 net load hours of the year give good CC approximations for solar photovoltaic (PV) resources (Stephen, Hale, and Cowiestoll 2020). At higher PV contributions, choosing the top 10 net load hours gave a closer approximation by 2\%-5\% (Stephen, Hale, and Cowiestoll 2020). Other case studies for both solar PV (with and without storage) and concentrating solar power in the United States find CF approximation methods to be close to full reliabilitybased methods (Madaeni, Sioshansi, and Denholm 2013, Madaeni, Sioshansi, and Denholm 2012, Mills and Rodriguez 2020, Heath and Figueroa-Acevedo 2018). Existing work evaluating CC calculations for wind energy is more sparse. However, a case study of wind in Portugal evaluates approximation-based and probabilistic methods and concludes that both calculation methods are useful, but chronological approximation methods might be better suited for system operators, whereas probabilistic methods are better suited for system planning (Castro and Ferreira 2001). Another evaluation of wind in Alberta finds that simpler CF approximation methods might overestimate the CC of wind in that system (Awara et al. 2018, Awara 2019).

Despite the fact that CC is a commonly used metric for resource comparison, it is not a perfect metric. Although it might provide an estimate of the amount of firm capacity a generator might provide, the actual capacity available during emergent times can vary considerably from the predicted value. As an example, a stand-alone PV plant could have a CC of $20 \%$; however, if capacity was needed during nighttime, it would not be available at all. In addition, $\mathrm{CC}$ is only one factor that might influence the deployment of various generator types (including wind), and it can often be lower priority than selecting high CF resource areas or transmission access, which can be important

\footnotetext{
${ }^{1}$ The terms capacity credit and capacity value are often used interchangeably in the literature; however, in this paper, we follow an established convention to refer only to physical capacity rather than monetary or economic value (Mills and Wiser 2012).

${ }^{2} \mathrm{Net}$ load is the demand less contributions from variable generation resources such as wind and solar.

${ }^{3}$ Other reliability-based methods such as convolution are not often amenable to transmission modeling.
} 
drivers to capital cost (Jorgenson et al. 2021). In short, calculating CC is a useful but imperfect means to incorporate weather-dependent resources into established resource adequacy assessment and integrated planning processes.

This work uses a Texas Interconnection test system to examine the impact of methods and assumptions on the resulting marginal wind CCs of both land-based and offshore wind for near-term deployment. Specifically, we compare reliability-based resource adequacy methods using the National Renewable Energy Laboratory's (NREL's) Probabilistic Resource Adequacy Suite (PRAS) model with approximation methods, along with methodological choices and parameters for both methods. First, for the reliability-based resource adequacy calculations, we consider the impact that the starting system assumptions have on the reported CC. Second, for the CF-based approximation methods, we examine the impact of considering the contributions of existing variable generation sources, varying the number of hours averaged, seasonal calculations, and transmission access assumptions. This paper can provide insight into how underlying assumptions can impact resulting wind CC by comparing across multiple CC calculation methods. 


\section{Methodology and Assumptions}

This analysis uses a case study to conduct a sensitivity on various CC calculation methods. We examine land-based and offshore wind in the Texas Interconnection test system, under the footprint of the Electric Reliability Council of Texas (ERCOT), which covers much of the state of Texas. We use the Texas Interconnection because it is an electrically isolated, relatively small power system with diverse resources for both wind and solar. In this section, we first detail the Texas Interconnection test system, then discuss the methodologies for using a probabilistic assessment of marginal wind CC with NREL's PRAS model as well as various approximation methods.

\subsection{Texas Interconnection Test System}

A full resource adequacy assessment of large power systems requires extensive data, including generator characteristics, transmission topology, and time-series profiles such as load. For this test system, we use generator data, load profiles, and existing renewable energy output time series for the Texas Interconnection test system (shown in Figure 1) from NREL's North American Renewable Integration Study (NARIS) using seven time-synchronous years of weather and load data (Brinkman et al. 2021). We derived the generation capacity for 2024 initially from NARIS, which includes $24 \%$ of total generation coming from wind and $12 \%$ from PV, before curtailment ${ }^{4}$. From that starting point, we removed generator capacity (mostly natural gas and coal units ${ }^{5}$ ) to create a system with observable shortfall, indicated by nonzero EUE (or curtailed load). We refer to this as our Base Case system, and Table 1 shows this fleet. This analysis does not include any planned or scheduled maintenance outages, which could reduce generator availability, particularly during the nonpeak (or shoulder) seasons ${ }^{7}$. As shown in Figure 1, the system has seven modeled zones within the Texas Interconnection test system. The numbers on the map correspond to the modeled balancing areas in NREL's Regional Energy Deployment System (ReEDS) (Brown et al. 2020). We include transmission capacity limits between these zones but not within.

\footnotetext{
${ }^{4}$ As of 2020 , wind generation comprised approximately $23 \%$ of total generation in the Texas Interconnection, with PV at approximately $2 \%$ (ERCOT Quick Facts February 2021).

${ }^{5}$ For our Base Case system, we removed approximately $19 \mathrm{GW}$ of capacity from the Texas Interconnection system. We removed generators with the lowest CFs and continued until we observed nonzero EUE ${ }^{6}$. Removing generator capacity inherently changes the system and might impact results, which is why we include variations on this Base Case in later sections.

${ }^{7}$ Typically planned maintenance would not occur during times of system need and is unlikely to impact the CC in this analysis. However, this shortcoming should be addressed in future work.
} 


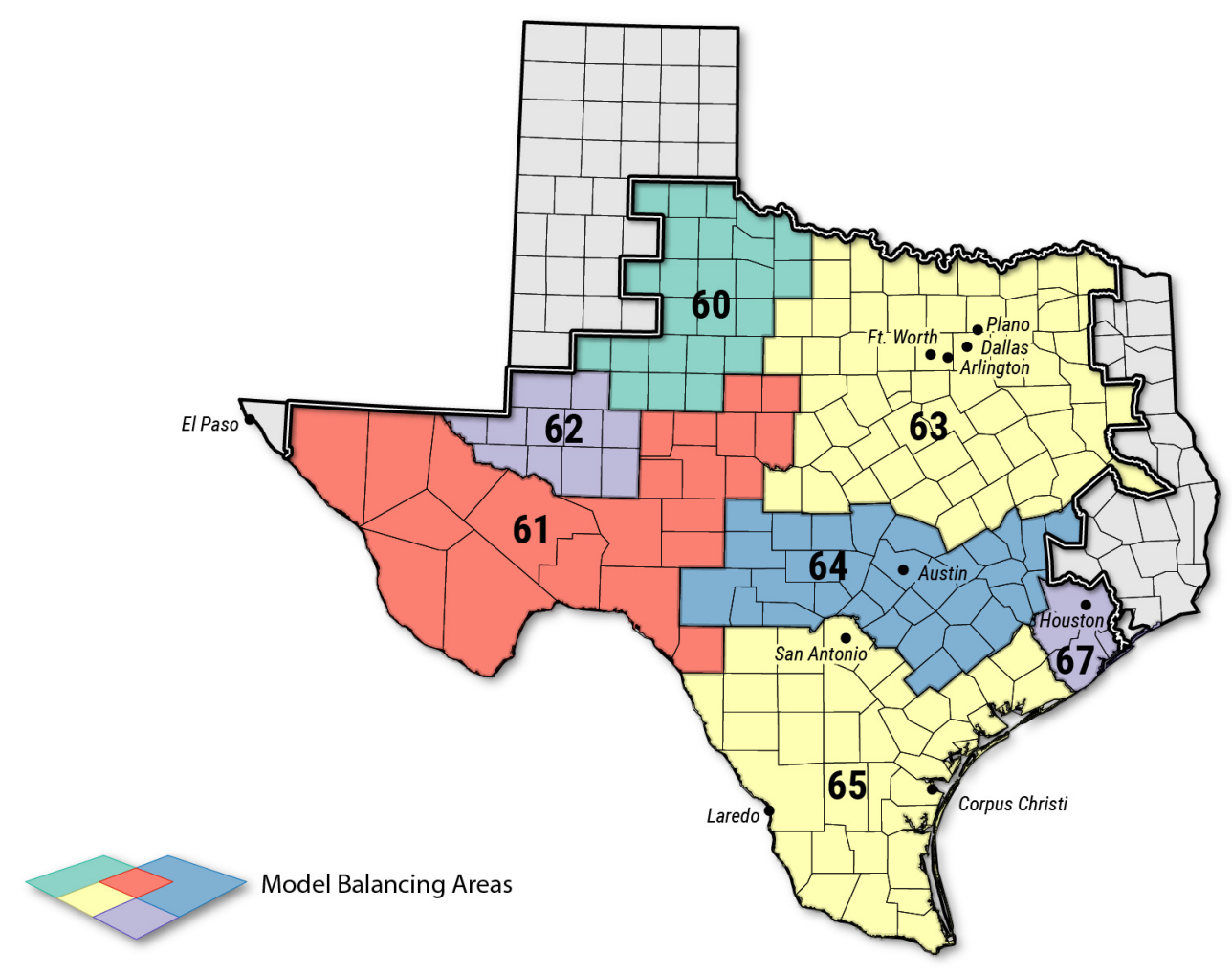

Figure 1. Map of the modeled footprint, including the seven modeled zones with numbers corresponding to the ReEDS modeled balancing areas (Brown et al. 2020).

\begin{tabular}{|l|c|}
\hline \multicolumn{2}{|l|}{ Modeled Generating Capacity by Type in the Texas Interconnection Test System } \\
\hline Wind & $24,000 \mathrm{MW}$ \\
PV & $23,400 \mathrm{MW}$ \\
Coal & $5,000 \mathrm{MW}$ \\
Natural gas & $50,400 \mathrm{MW}$ \\
Nuclear & $5,000 \mathrm{MW}$ \\
Other $^{8}$ & $550 \mathrm{MW}$ \\
Total & $\mathbf{1 0 8 , 0 0 0} \mathbf{M W}$ \\
\hline \multicolumn{2}{|c|}{ Demand Characteristics } \\
\hline Peak hourly load & $75,000(7-\mathrm{yr}$ Range: 72,800-75,000) MW \\
Peak hourly net load & 62,800 (7-yr Range: 59,700-62,800) MW \\
Average annual load & $365 \mathrm{TWh} / \mathrm{yr}$ \\
Average annual net load & $224 \mathrm{TWh} / \mathrm{yr}$ \\
\hline
\end{tabular}

Table 1. Assumptions for the modeled Base Case generating capacity by type, along with system demand.

The hourly profiles for existing wind, utility-scale PV, and distributed PV are from the Wind Integration National Dataset (WIND) Toolkit (Draxl et al. 2015) and the National Solar Radiation Database (Sengupta et al. 2018), processed through NREL's System Advisor Model (Blair et al. 2018) with specific technology assumptions to generate

\footnotetext{
${ }^{8}$ Includes hydropower, storage, and biofuel.
} 
CF profiles (Maclaurin et al. 2019). For representing new wind resource, we also use the WIND Toolkit, which contains wind data for 2-km by 2-km grid cells for this footprint over 7 weather years (Draxl et al. 2015); however, to reduce computational demands and to more closely match the footprint of modern wind power plants, we aggregated profiles to a resolution of $20 \mathrm{~km}$ by $20 \mathrm{~km}^{9}$ (Jorgenson et al. 2021). We consider two turbine types: one technology for land-based sites and one for offshore sites. Table 2 shows the technology specifications we use to generate the profiles for the incremental wind (Jorgenson et al. 2021) ${ }^{10}$. We employ 7 years of system load, wind, and solar profiles (2007 through 2013). Using multiple years of weather data more fully captures the impact of interannual variability, as is recommended for a robust analysis of CC (Milligan 1997, Keane et al. 2010, Milligan et al. 2017). Figure 2 shows the geographic footprint with the 7 -year average CF for wind at all of the $20-\mathrm{km}$ by $20-\mathrm{km}$ grid cells, indicating both land-based and offshore sites. The map shows particularly high CFs for central Texas as well as offshore sites in the Gulf of Mexico. Although there is currently no operating offshore wind in the Gulf of Mexico, there is interest in future development (Musial et al. 2020).

\begin{tabular}{|l|c|c|}
\hline Technology & Land-Based & Offshore \\
\hline Rotor diameter $(\mathrm{m})$ & 108 & 222 \\
Capacity $(\mathrm{MW})$ & 2.1 & 12 \\
Hub height $(\mathrm{m})$ & 80 & 136 \\
Specific power $\left(\mathrm{W} / \mathrm{m}^{2}\right)$ & 229 & 310 \\
Swept area $\left(\mathrm{m}^{2}\right)$ & 9160 & 38700 \\
Turbine losses $(\%)^{11}$ & 16.7 & 16.7 \\
\hline
\end{tabular}

Table 2. Turbine specifications used to generate the CF profiles for the incremental wind capacity.

\footnotetext{
${ }^{9} \mathrm{Here}$, we evaluate all grid cells without screening for other economic or suitability factors. This includes cells with existing wind turbines and those without.

${ }^{10}$ Note that the technology specifications in Table 2 do not necessarily represent installed wind turbines in the Texas Interconnection. Since we are evaluating incremental wind capacity, we use these parameters as a reasonable proxy for the characteristics wind turbines being installed currently.

${ }^{11}$ Here, turbine losses are applied as a fixed percentage in every hour, which is a shortcoming since in some hours the losses would be much less (or perhaps even greater). Future work should evaluate the drivers of turbine losses.
} 


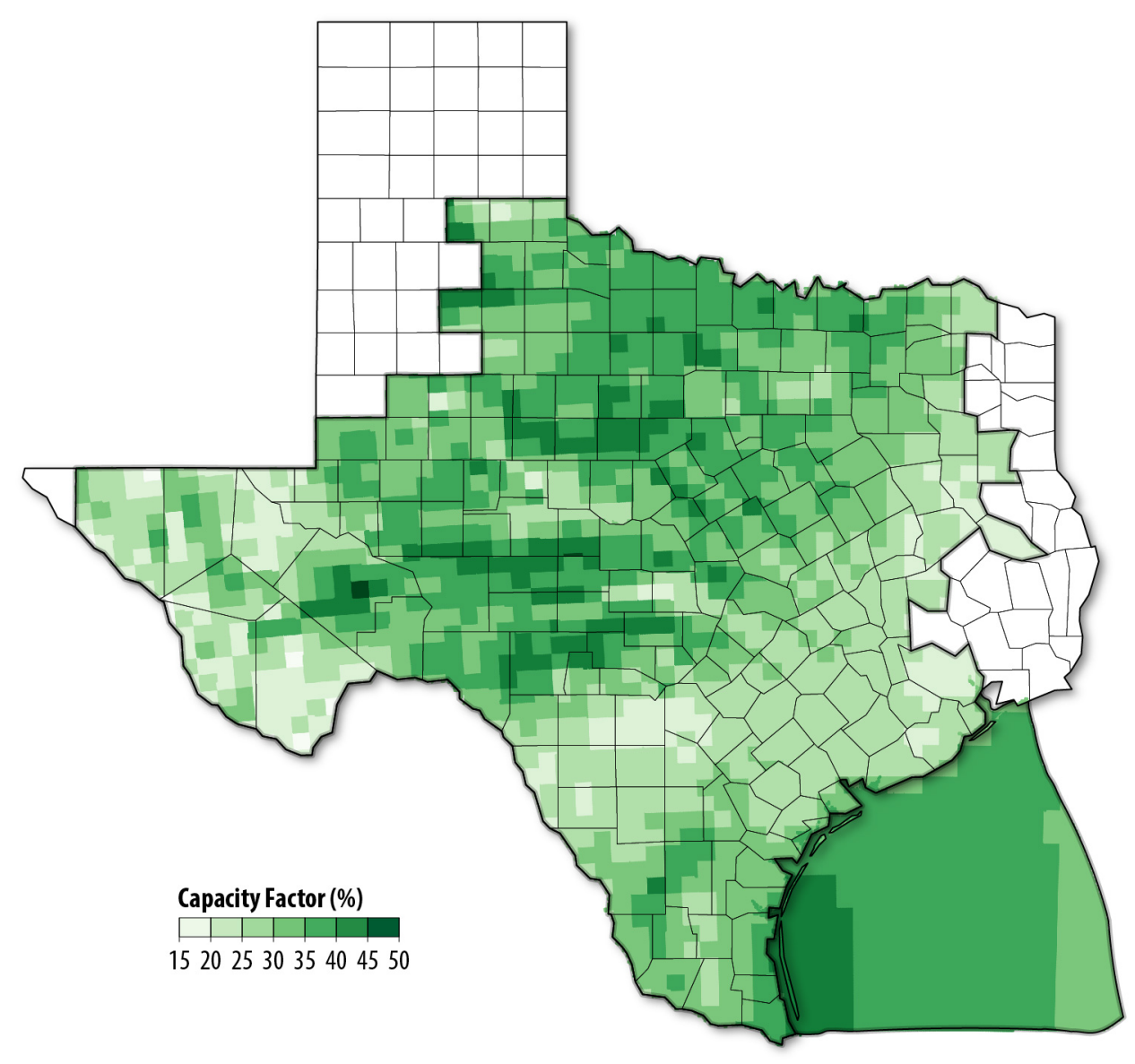

Figure 2. Map of the 7-year average CF calculated from WIND Toolkit data for 2007-2013.

Figure 3 illustrates the 7-year average hourly diurnal demand curves (top panel) and net load (bottom panel) for each month. The solid line indicates the average observation, with the orange ribbons indicating the observed percentiles. The peak demand occurs in the summer months, in the late afternoon or evening. The lightest orange ribbons ( 0 100) indicate that there is significant variation in demand in some months, especially January and February. The net load shows even wider ranges in many months because of the additional variation imposed by the wind and solar resources. The solar and wind resources reduce the net load (relative to the gross load), particularly in the middle of the day, when solar availability is the highest. Peak net load is lower than peak load, but it still occurs generally in the summer, though in some cases it is later in the day. On some days, the orange ribbons indicating computed net load actually drop below $0 \mathrm{GW}$, indicating that available solar and wind exceed demand ${ }^{12}$.

${ }^{12}$ The Texas Interconnection test system has 23\% wind and 12\% PV contribution before curtailment. Many hours have substantially more (or less) than that. 


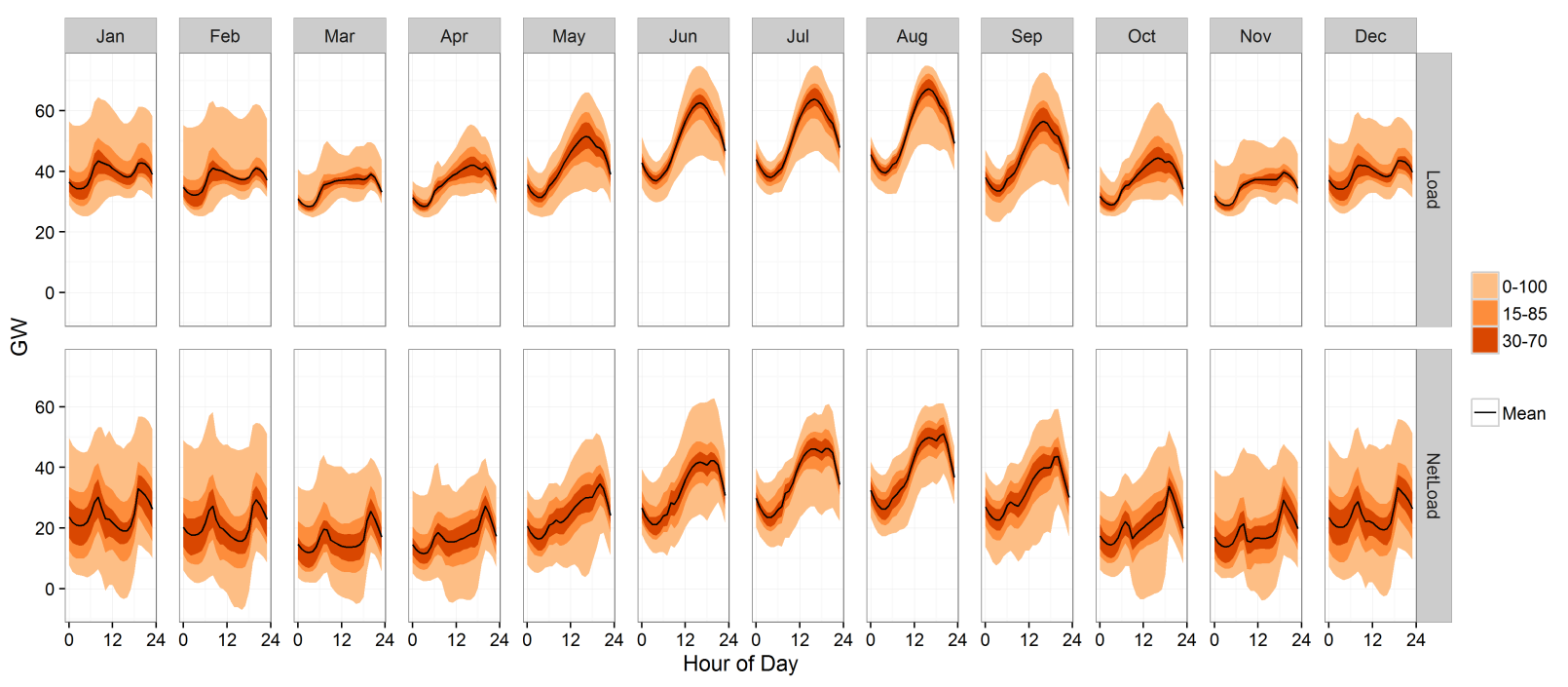

Figure 3. Average diurnal load and net load profiles by month for the Texas Interconnection test system, with ribbons indicating the observed percentiles over the 7 years of data (2007-2013). This reflects our Base Case generator fleet, with $24 \%$ generation contribution from wind and $12 \%$ from solar.

Figure 4 illustrates the average 7-year diurnal wind CF curves for each month of the year. The black line indicates the average observation based on all years and $20-\mathrm{km}$ by $20-\mathrm{km}$ sites. The green ribbons indicate the observed percentiles ${ }^{13}$. First, we note that both land-based and offshore wind exhibit a typical diurnal curve in which wind generation generally decreases in the middle of the day and is highest overnight. This midday reduction in wind CF in Texas has been observed previously (Musial et al. 2020, AWS Truepower 2015). Second, we note that offshore wind (the top panel) has overall higher CFs during the non-summer months and is more consistent throughout the course of the day than land-based wind; however, offshore wind output is significantly reduced in the summer months (June-August). In fact, on average it shows a lower CF than land-based wind during those months, especially in the evening and early morning hours.

\footnotetext{
${ }^{13}$ The hourly CF never exceeds $83 \%$ because of turbine losses, which are applied as a fixed percentage in every hour, as noted in Table 2.
} 


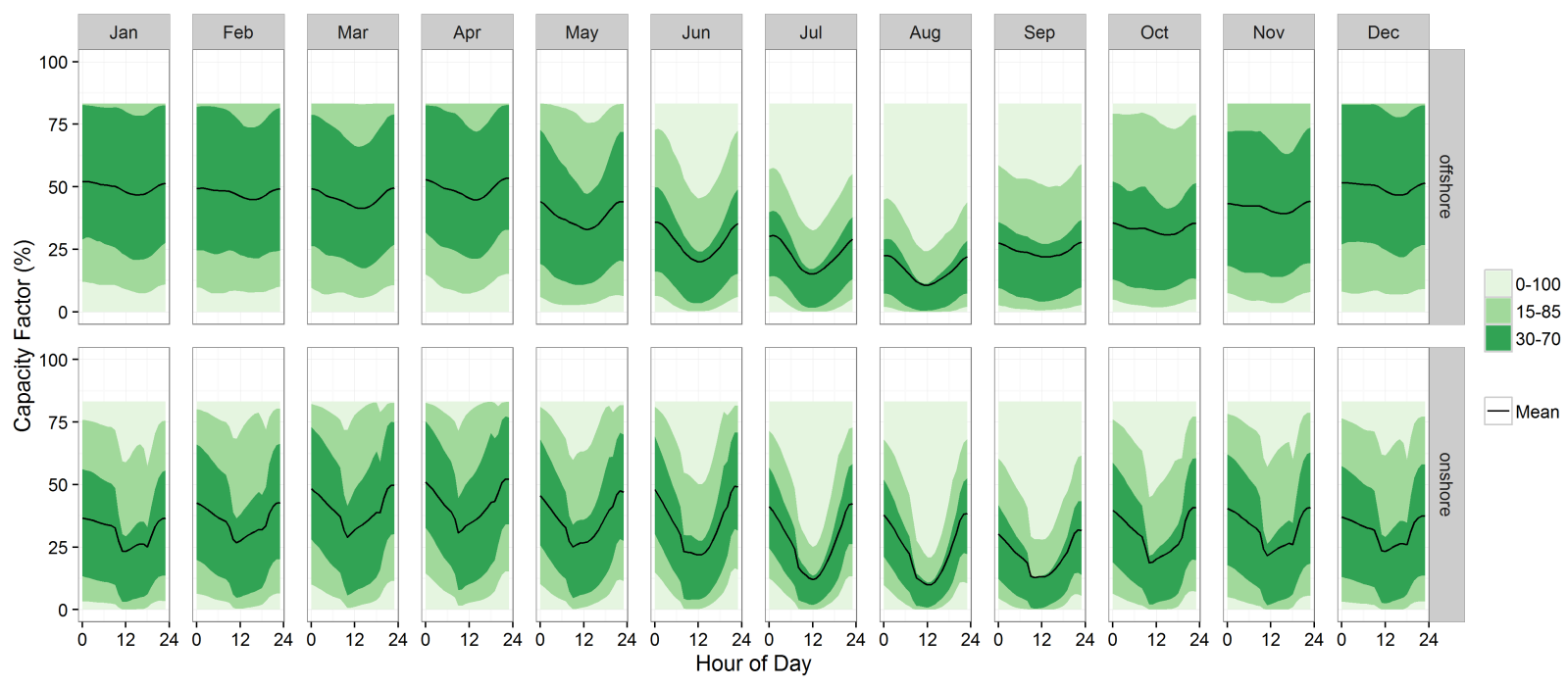

Figure 4. Average diurnal wind CFs from the 7 years of WIND Toolkit data for all grid cells, with ribbons indicating the observed percentiles.

\subsection{Methods}

Probabilistic resource adequacy tools quantify the risk of being unable to serve demand at all times with metrics such as EUE or normalized expected unserved energy (NEUE). ${ }^{14}$ These tools analyze the distribution of possible system states, given the probability of unit outages, generation from variable generation resources such as solar and wind, and demand. Using random sampling, probabilistic resource adequacy models assess a large number of possible system states and calculate these probabilistic reliability metrics. To conduct a probabilistic resource adequacy analysis, we apply NREL's Probabilistic Resource Adequacy Suite (PRAS) tool (Stephen 2019). PRAS uses interregional power transfer constraints and Monte Carlo draws from probability distributions for generator availability to calculate the loss of load resulting from supply shortfall or transmission constraints ${ }^{15}$ (Beiter et al. 2020, Frew et al. 2019, Stephen, Hale, and Cowiestoll 2020, Stephen 2021). For each hour, we use 100,000 Monte Carlo draws of forced generator outages to compute the EUE for a given level of net load ${ }^{16}$, which are aggregated into annual values. As discussed, we created our Base Case (Table 1) to have nonzero EUE. Then, we express CC using a common reliability-based metric, equivalent firm capacity (EFC) (Soder and Amelin 2008). EFC expresses the CC of the incremental wind in terms of a 100\% reliable (fully firm) unit. We use EUE as the probabilistic resource adequacy metric to quantify system risk to capture the impact of the new resource in reducing both the magnitude and the duration of shortfalls. For example, if a 100-MW wind installation reduces the EUE by $100 \mathrm{GWh}$ and a $45-\mathrm{MW}$ firm capacity unit also reduces the EUE by $100 \mathrm{GWh}$, the CC of the $100-\mathrm{MW}$ wind farm is $45 \mathrm{MW}$, or $45 \%$. We calculate the system-wide EUE during 7 years of operations, before and after adding wind to each grid cell. We then determine the level of firm capacity that, when added to the same region, would result in an identical reduction in system-wide EUE. The firm capacity divided by the incremental rated capacity of wind (in this case, $500 \mathrm{MW}$ per cell) gives the CC.

In this analysis, we evaluate the impact of assumptions and methods on the resulting marginal CCs. First, we compare various system cases in our probabilistic resource adequacy tool (PRAS) to assess the starting criteria on resulting CC, and we compare the results to our original Base Case, shown in the first column of Table 3 and discussed in Section 3. Then, we further compare this Base Case PRAS-calculated CC to various CF approximation methods to explore what might be lost or gained from using these simpler approximation methods, shown on the right four

\footnotetext{
${ }^{14} \mathrm{NEUE}$ is the total EUE, typically measured in MWh or GWh, divided by the total load (also in MWh or GWh), often reported in terms of parts per million (ppm).

${ }^{15}$ For this analysis, we assume that the transmission links between modeled zones are $100 \%$ reliable.

${ }^{16}$ The net load is a deterministic value for each hour in the seven year set.
} 
columns of Table 3, discussed in Section 4.

\begin{tabular}{|c|c|c|c|c|}
\hline \multirow{2}{*}{ Sensitivities on PRAS CC } & \multicolumn{4}{|c|}{ Sensitivities on CF-Approximation Methods } \\
\cline { 2 - 5 } & Impact of VG & Impact of No. of Hours & Impact of Transmission & Impact of Seasonality \\
\hline Original fleet (Base Case) & Top load hours & Top 1 hour & Region net load & CC by season \\
Double Winter FOR & Top net load hours & Top 10 hours & System net load & \\
Scale Load & & Top 100 hours & & \\
Remove Extra 3 GW & & Top 1000 hours & & \\
\hline
\end{tabular}

Table 3. Sensitivities of the starting point for PRAS-calculated CC and on CF approximation methods explored here. 


\section{Sensitivities on PRAS CC}

As discussed in the previous section, reliability-based resource adequacy calculations rely on many modeling decisions. These modeling decisions might or might not have a dramatic impact on the resulting resource adequacy metrics (and thus marginal wind CC assessed here). First, we compare various system cases in PRAS to assess the starting criteria on the resulting CC. As mentioned, computing the $\mathrm{CC}$ of a marginal resource requires first creating a system with nonzero EUE; however, the route to creating a system with nonzero EUE is not always clear. So, we create variations to see how the decisions involved in creating the Base Case impact the results. We first consider a case with a doubled generator forced outage rate in the winter months (December through February) because our starting case uses a constant year-round value for outage rates, whereas in reality generators often have increased forced outage rates in cold temperatures (Murphy, Lavin, and Apt 2020, Murphy, Sowell, and Apt 2020). We also consider a sensitivity in which we uniformly scale load by a factor of 1.26 in each hour, rather than remove generator capacity, which achieves the same goal of creating nonzero EUE. One consideration of this method is that scaling load without increasing the capacity of transmission lines changes the relative amount of power that can be exchanged between zones and thus can impact the estimations for CC, particularly for zones that rely on imports. In addition, scaling load would not necessarily account for changes in the load shape, which could be driven by diffusion of technologies such as electric vehicles or heating. Last, we evaluate the sensitivity to the initial generator fleet in Table 1 by removing $3 \mathrm{GW}$ of additional thermal generation capacity, resulting in a higher level of observed shortfall.

PRAS identifies periods when the system is the most stressed and generator capacity is the most essential. These times of system stress determine the periods that will most heavily influence the CC for marginal generatorsin this case, wind. Figure 5 illustrates the average diurnal system EUEs for each month of the 7 years considered here. Each row represents one PRAS formulation in the first column of Table 3. The figure demonstrates that for all years and sensitivities, the highest levels of EUE occur during the summer in the late afternoon or early evening. Historically, this is when many parts of the country, including Texas, see peak demand. For much of the United States, hot summer temperatures lead to sizeable air-conditioning load, which, when combined with other electricity use, results in peak electricity demand ${ }^{17}$ ). Generators that are available to produce electricity in these periods can help reduce the EUE and will exhibit higher CC. The availability of the wind during periods of EUE, subject to zonal transmission constraints, will determine the marginal CC of wind. We note that Figure 5 shows similar times of EUE for each scenario that we evaluate here. Although the EUE can vary in terms of magnitude across scenarios, the same pattern emerges for each PRAS sensitivity

\footnotetext{
${ }^{17}$ Not all regions of the United States experience peak demand in the summer, especially regions with more temperature summers. Extreme storms and cold weather events can also impact when peak demand occurs (The Electric Reliability Council of Texas 2021).
} 


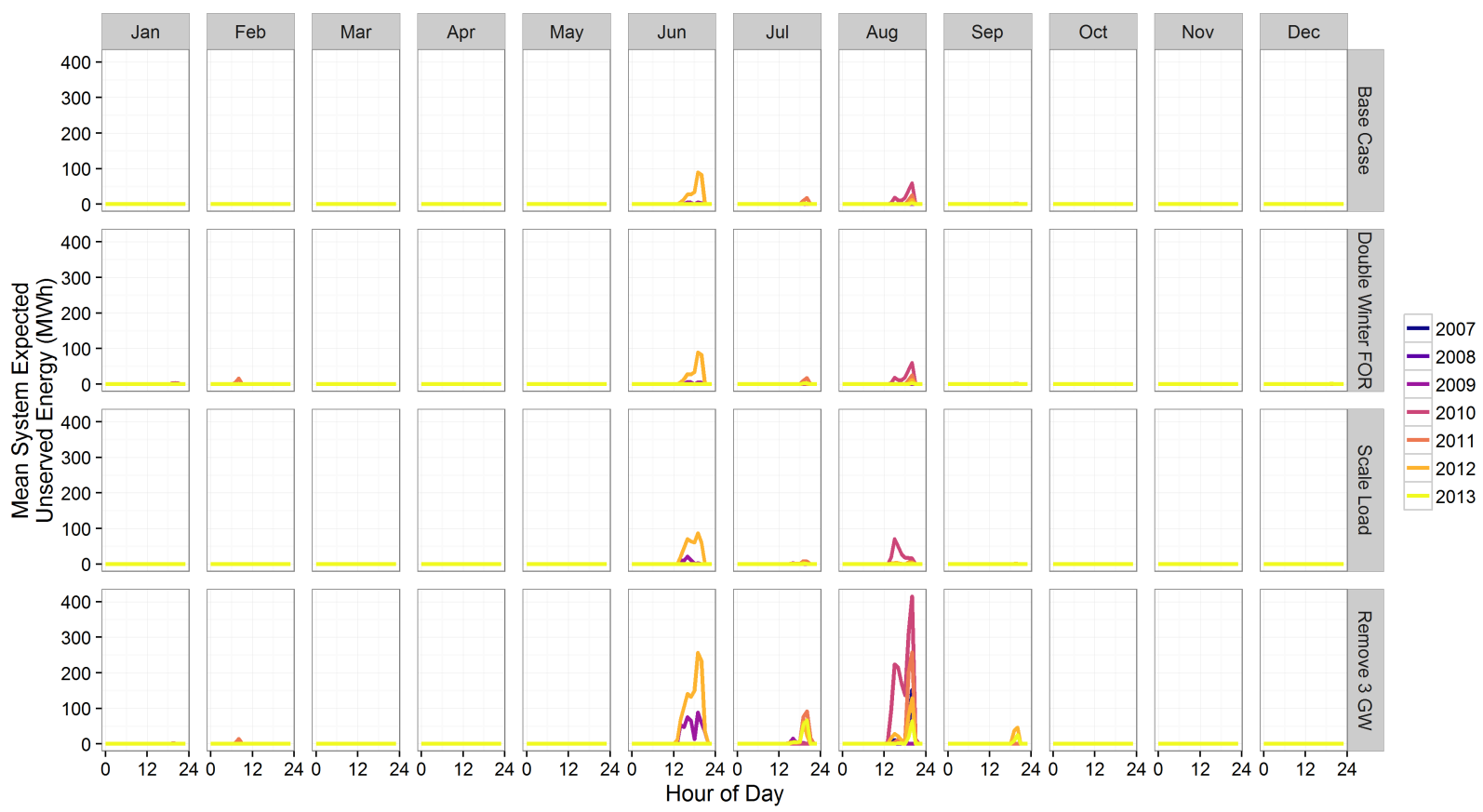

Figure 5. Average diurnal system-wide EUE calculated by PRAS for the 7 years studied here by month, time of day, and scenario.

Figure 6 shows the resulting CCs for the Base Case. The CC varies by location, but the simple average $\mathrm{CC}$ for all land-based cells in the footprint is $12.7 \%$ (ranging between $4 \%$ and $42 \%$ ) and for average offshore is $6.7 \%$ (ranging between $2 \%$ and $27 \%$ ). Generally, the average $\mathrm{CC}$ is relatively small compared to other regions of the United States, particularly for offshore wind (Jorgenson et al. 2021, Beiter et al. 2020, New England Wind Integration Study 2010, Dvorak et al. 2013). Notably, the Texas Interconnection test system already has substantial deployment of land-based wind technology, and sites are generally well correlated because of the smaller footprint compared to the other U.S. interconnections. As discussed, the wind availability in the evening during the summer (when shortfalls tend to occur) is lower than the average wind availability, and it is even lower for offshore wind than for land-based, leading to generally low CCs. For example, the CF for the average offshore wind cell for the month of August (when most EUE occurs in this system) is $40 \%$ lower than the average August CF for land-based sites. 


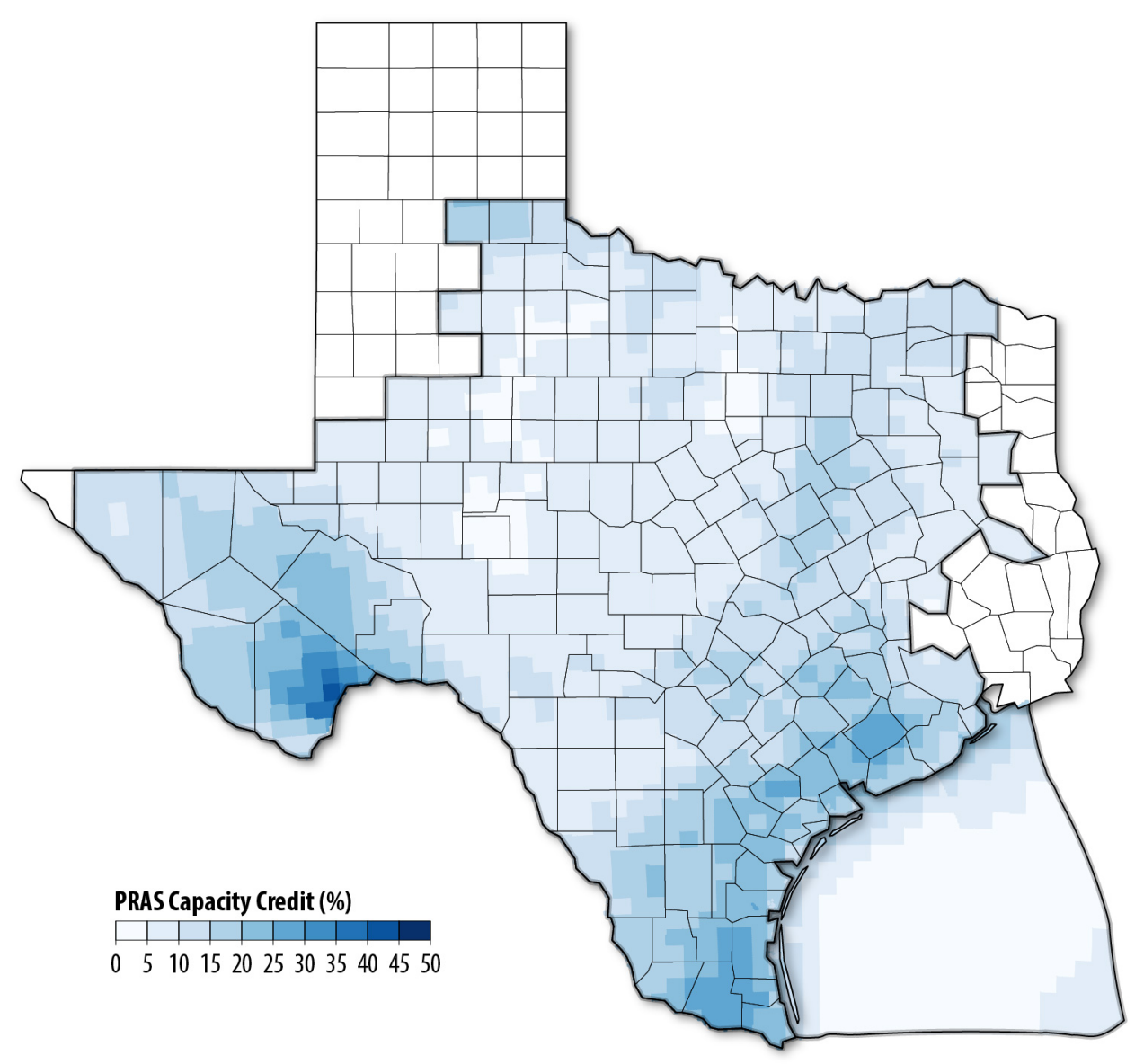

Figure 6. Base Case PRAS-computed marginal CC for each grid cell in the footprint.

Figure 7 shows the difference between the Base Case PRAS-computed marginal CC for each grid cell compared to three different variations on the starting PRAS case. A positive value indicates that the new starting point increased the marginal CC, whereas a negative value represents a reduced CC. Across the Texas Interconnection footprint, different starting point plots show mild differences in terms of overall of wind CCs, which generally agrees with the observation from Figure 5, which showed similar times of system need across scenarios. For example, the overall average marginal CC for wind in the Base Case is $11.7 \%$, whereas it is $12.4 \%$ in the Double Winter FOR Case, $8.8 \%$ in the Scale Load scenario, and $11.9 \%$ in the Remove 3 GW Case. 

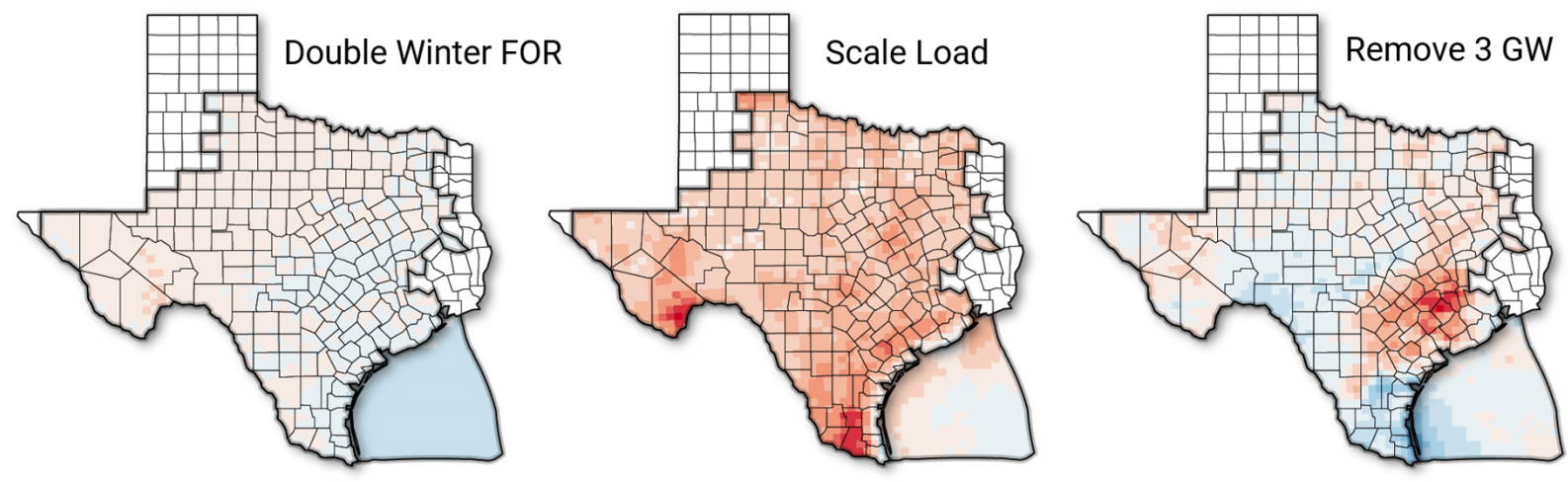

Capacity Credit Difference (\% points)

$\begin{array}{lllllllllllll}-15-12-10 & -8 & -6 & -4 & -2 & 0 & 2 & 4 & 6 & 8 & 10 & 12 & 15\end{array}$

Figure 7. The difference between the Base Case PRAS-computed marginal CC for each grid cell compared to three different variations on the starting PRAS case. A positive indicates the new starting point increased the marginal $\mathrm{CC}$, whereas a negative value represents a reduced $\mathrm{CC}$.

However, the map does indicate more significant changes to specific grid cells or regions. The Scale Load sensitivity generally indicates lower CCs than the generator removal technique indicated in the Base Case. In this sensitivity, the load is scaled by a factor of 1.26 in each hour ${ }^{18}$. The increased load puts additional stress on the transmission lines, leading to more congestion. That congestion ties up the transmission lines, which slightly reduces the ability of wind generation to be able to effectively reduce EUE; however, the impact is still relatively minor and does not dramatically change the $\mathrm{CC}$ magnitudes or geographic patterns. But this impact could become even more pronounced in regions or scenarios with greater load scaling for nonzero EUE.

In the Double Winter FOR case, the CC of wind increases from $12.7 \%$ for land-based to $13.0 \%$, and from $6.7 \%$ for offshore to $9.8 \%$. Figure 5 shows that the EUE remains the same in the summer months and slightly increases in the winter months. The slightly increased risk in the winter months favors wind generation patterns (Figure 4), leading to a slightly higher CC, especially for offshore wind; however, the impact is relatively minor because the vast majority of EUE still occurs in the summer months.

Last, the Remove $3 \mathrm{GW}$ case shows very little difference in terms of overall average (11.9\% compared to $11.7 \%$ in the Base Case). Most grid cells on the map show small differences compared to the Base Case except for a red region in zone 64 located geographically between Austin and Houston. For these dozen grid cells, the change in the timing of the EUE (Figure 5) heavily weights the importance of hours when wind production (for that specific geographic region) is less favorable, leading to a very localized negative impact on wind CC. For most grid cells, however, the impact is not dramatic and does not change the overall picture of wind CC in the Texas Interconnection test system.

Overall, the map of these various starting points for the PRAS CC calculation actually illustrates more similarities than differences in terms of calculated CCs, and the main conclusion remains constant across these scenarios: the $\mathrm{CC}$ for wind in this Texas Interconnection test system is relatively low, and it is even lower for offshore wind than land-based. However, the sensitivities do indicate some limitations for the CC metric generally, since transmission constraints do impact wind $\mathrm{CC}$ and including those constraints is not always straightforward. We discuss these trade-offs with more detail in the following section.

\footnotetext{
${ }^{18}$ We chose the 1.26 scaling factor since it resulted in a similar level of EUE compared to the Base Case scenario.
} 


\section{Comparisons with Approximation Methods}

As previously discussed, approximation methods for calculating the $\mathrm{CC}$ of marginal resources, including wind, are widely used, in large part because of the relative simplicity of the methods compared to reliability-based approaches such as PRAS. Similar to resource adequacy models, however, there are many criteria and considerations that can potentially impact the resulting CC. In this section, we first compare the approximation-based methods to the reliability-based method (in this case, the Base Case scenario using PRAS). Then, we explore the sensitivity of the resulting wind $\mathrm{CC}$ to various chosen approximation methods, as shown in the right four columns of Table 3. In most of these approximation-based methods, the $\mathrm{CC}$ is estimated by calculating the average $\mathrm{CF}$ during key time periods, chosen to represent times of system stress. These approximation methods are widely used with various assumptions (Stephen, Hale, and Cowiestoll 2020, Mills and Rodriguez 2019, Cole et al. 2020). We choose these key periods with multiple selection criteria to compare across various methodologies typically encountered. First, we evaluate the impact of transmission by calculating a CF approximation with either the "regional" criteria (that is, for each of the seven modeled zones) or the overall "system" criteria (for the Texas Interconnection test system as a whole). For this approximation, we use a weighted CF approximation, which weights each hour based on the EUE experienced each hour. Second, we examine the impact of variable generation (VG) by comparing the CC calculated as the simple average for the top load hours to the top net load hours. Third, we examine the impact of the numbers of hours included in the $\mathrm{CF}$ approximation by evaluating the $\mathrm{CC}$ by again taking the simple average of the CF during the top 1,10,100, and 1000 hours each year. Fourth, we evaluate the impact of seasonality by calculating the $\mathrm{CF}$-approximated $\mathrm{CC}$ during each season rather than the average for the whole year.

\subsection{Computed with EUE-Weighted CF}

First, we examine a CF approximation method that considers the hourly EUE profiles shown in Figure 5. In the previous section, we use PRAS to assess the improvement in EUE with the incremental wind, thus giving the CC for each grid cell. For this approximation method, instead of using PRAS to compute the CC for each grid cell, we approximate by comparing the hourly CF profiles for each grid cell with the hourly EUE. We simply weight the hourly CF by the EUE time series. This method still relies on PRAS to generate the initial hourly EUE profiles, but it does not require running PRAS for each grid cell representing incremental wind.

We compute these CC approximations under two assumptions: one where we compute the EUE-weighted CF using the Texas system-wide hourly EUE profile and one where we compute the EUE-weighted CF based on the regional EUE time series corresponding to the zones shown in Figure 1. Figure 8 shows these CC approximations plotted against the PRAS-computed CCs in the Base Case formulation, with the corresponding difference maps shown in Figures 9. Initially, we note much tighter agreement for the system EUE-weighted approximation for both landbased and offshore wind sites; however, the system EUE approximation generally slightly overestimates the CC because this method assumes that wind at each grid cell can address EUE anywhere, whereas the PRAS-computed $\mathrm{CC}$ includes regional transmission constraints that could restrict CC. The right side of Figure 9 plots the difference between the PRAS estimate and the system EUE-weighted CF on the map, which shows relatively small differences.

The zone EUE-weighted approximation (on the left side of Figure 9) results in a wider scatter, however, where the approximation both over- and underestimates the PRAS-estimated CC, depending on the relative contribution of each system to the Texas system-wide EUE. This method assumes that incremental wind can address only shortfall within its own modeled zone. Key to understanding this result is the fact that this calculation assumes that wind in one region will not be able to address shortfall in another region, and the seven modeled zones do not experience EUE uniformly. In fact, zone 63 experiences $79.2 \%$ of the modeled EUE, and zone 61 experiences $17.3 \%$, with the other $3.5 \%$ split between the remaining four zones. So, zones 63 and 61 show region EUE-weighted CCs that look very similar to the system EUE-weighted CCs, whereas the calculation for the rest of the regions ends up highly weighting hours that turn out to be less important when considering the entire system. 

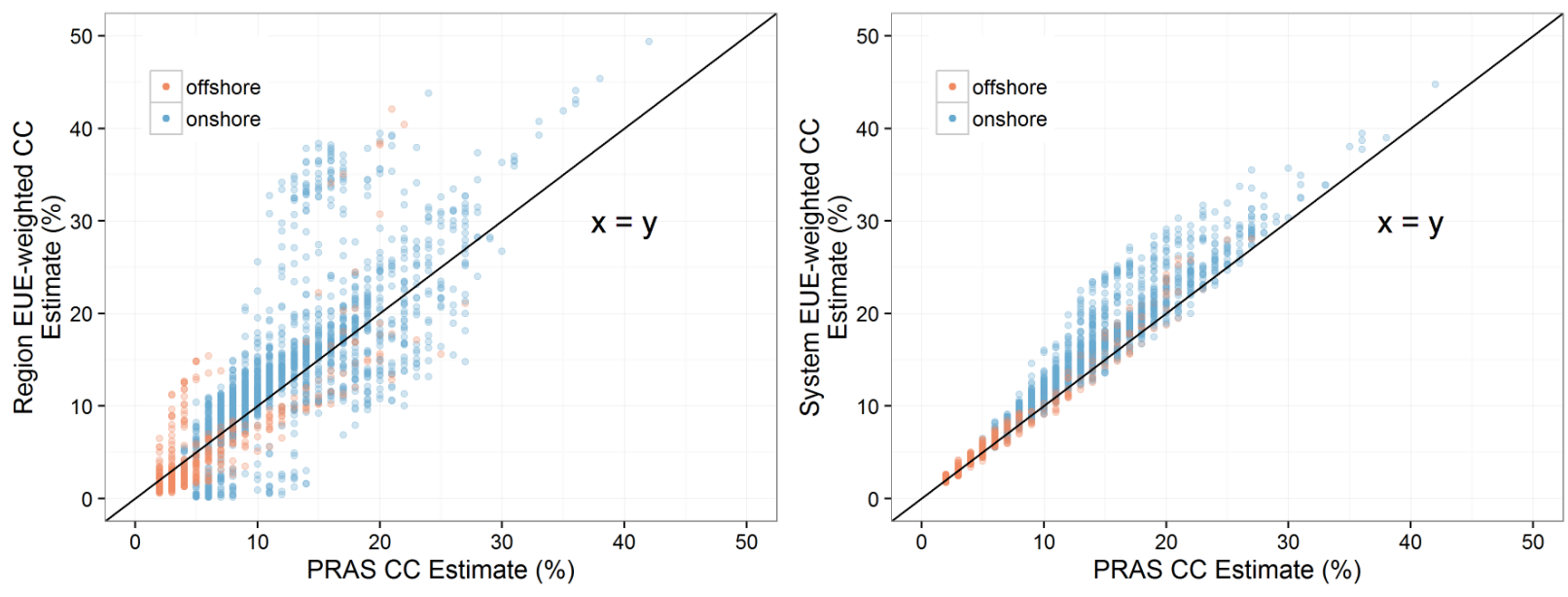

Figure 8. Comparison of zone EUE- and system EUE-weighted CF to the Base Case PRAS-computed CCs.
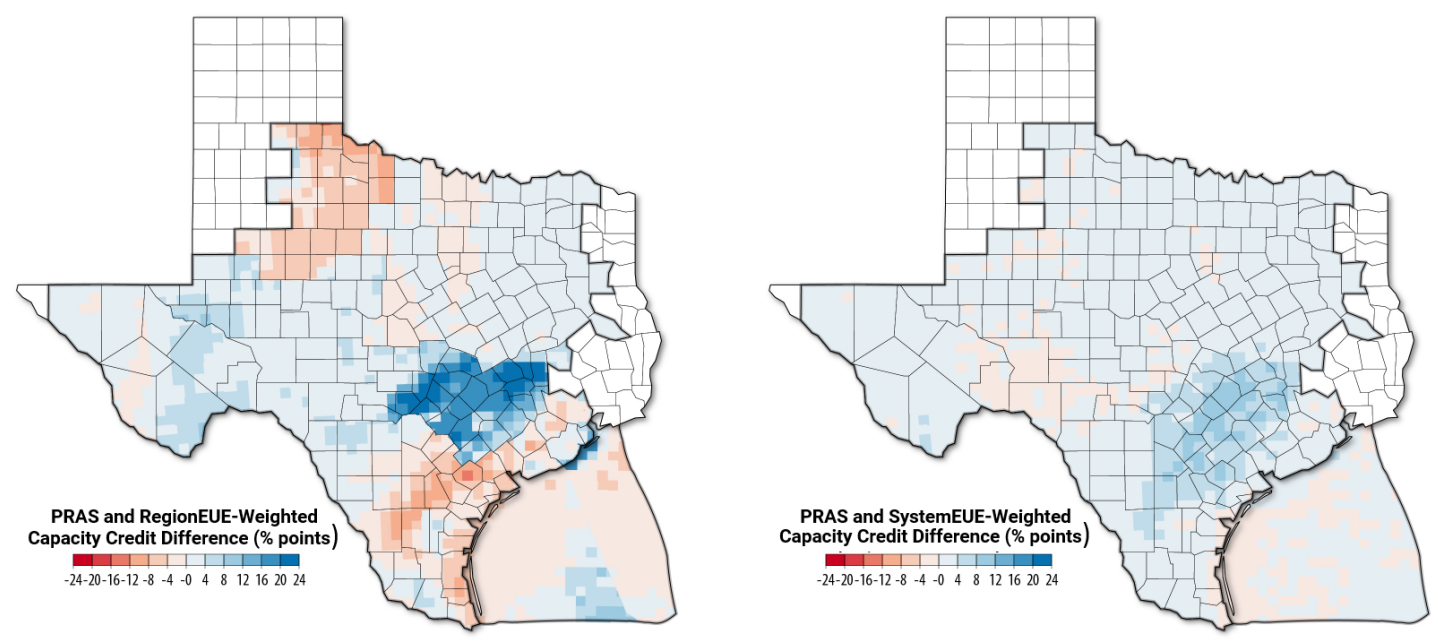

Figure 9. The difference between the $\mathrm{CC}$ approximation calculated by the CF weighted by either the Texas system-wide EUE or zone EUE and the Base Case PRAS CC.

Although these two approximation methods give somewhat similar average CCs (13.1\% for the system EUEweighted $\mathrm{CF}$ and $12.8 \%$ for the zone EUE-weighted CF, compared to the PRAS-computed average CC of 11.7\%), Figures 8 and 9 indicate a much better agreement between PRAS and the Texas system-wide EUE-weighted CF approximation method despite slightly overstating the CCs due to the omission of transmission constraints. This underscores the importance of the proper consideration of transmission constraints when evaluating the marginal CC of wind resources. For example, for transmission-constrained systems, considering zone-specific EUE will give a better picture of wind's CC, but in a system that is not limited by transmission, the system EUE calculation might give a better estimate. Further, this methodology does not consider intra-regional constraints, which might inhibit CC even further in specific locations.

\subsection{Estimated By Measures of Load}

Other commonly used CF approximation methods for CC rely on quantifying times of peak system load or net load, which generally are most likely to encounter shortfall conditions. These methods often choose a set number 
of hours per year (such as 10 or 100 hours) and then average CF during those hours to approximate CC for marginal variable resources. In this section, we compare various $\mathrm{CF}$ approximation methods using gross load and net load. We examine sensitivities on the number of hours chosen and the impact of considering regional (here, modeled zones) or system (here, total Texas Interconnection test system) load.

Figure 10 compares the $\mathrm{CC}$ computed with measures of load to the CC computed by the Base Case in PRAS. The four panels show CC computed by averaging the CF during the top 10 hours per year for each of the 7 years, either using load or net load (load less variable generation resources), and also comparing overall system load/net load to regional load/net load. Of the four methods depicted, Figure 10 shows the smallest spread in scatter plot points between PRAS and the CF approximation method that uses the overall system net load (top left). However, this method (using overall system net load) also tends to consistently overestimate the CC because it gives no consideration to transmission constraints, resulting in a higher estimation for CC compared to PRAS (16\% compared to $12 \%$ ).

Comparing the regional net load to PRAS (upper right panel of the plot) results in significant disagreement between PRAS and the estimate, despite the overall average site CC being similar (12\% for PRAS, $10 \%$ for regional net load calculation). The regional net load calculation method tends to underestimate the $\mathrm{CC}$ of wind because it does not capture the ability of the incremental wind to contribute to reducing net load in other regions.

Although the system has more wind than solar, solar's more predictable diurnal shape tends to shift the peak net load to later in the evening (as shown in Figure 3, comparing load and net load shapes). This slightly favors the wind profiles, which tend to increase later in the evening. Although Figure 10 has considerable spread comparing system load, the average CF during the highest load hours actually tends to underestimate the overall wind CC compared to PRAS (10\% compared to $12 \%)$.

The bottom two scatter plots (system versus regional load-estimated CC) are very similar to each other. The demand shape is quite uniform across regions, meaning that each region bears roughly the same shape as the total system load, but the same cannot be said for net load. That is because there is a significant regional variation in variable generation contribution (ranging from $0 \%$ wind penetration relative to load in zone 67 to $600 \%$ wind penetration in zone 60 , and from $3 \%$ solar in zone 63 to $166 \%$ in zone 60 ). So, considering the regional versus system net load (top two panels) is an important distinction, whereas considering regional versus system native load is not. This conclusion is specific to the Texas Interconnection test system, and in a system with more load diversity, then considering regional variation in load might become important. 


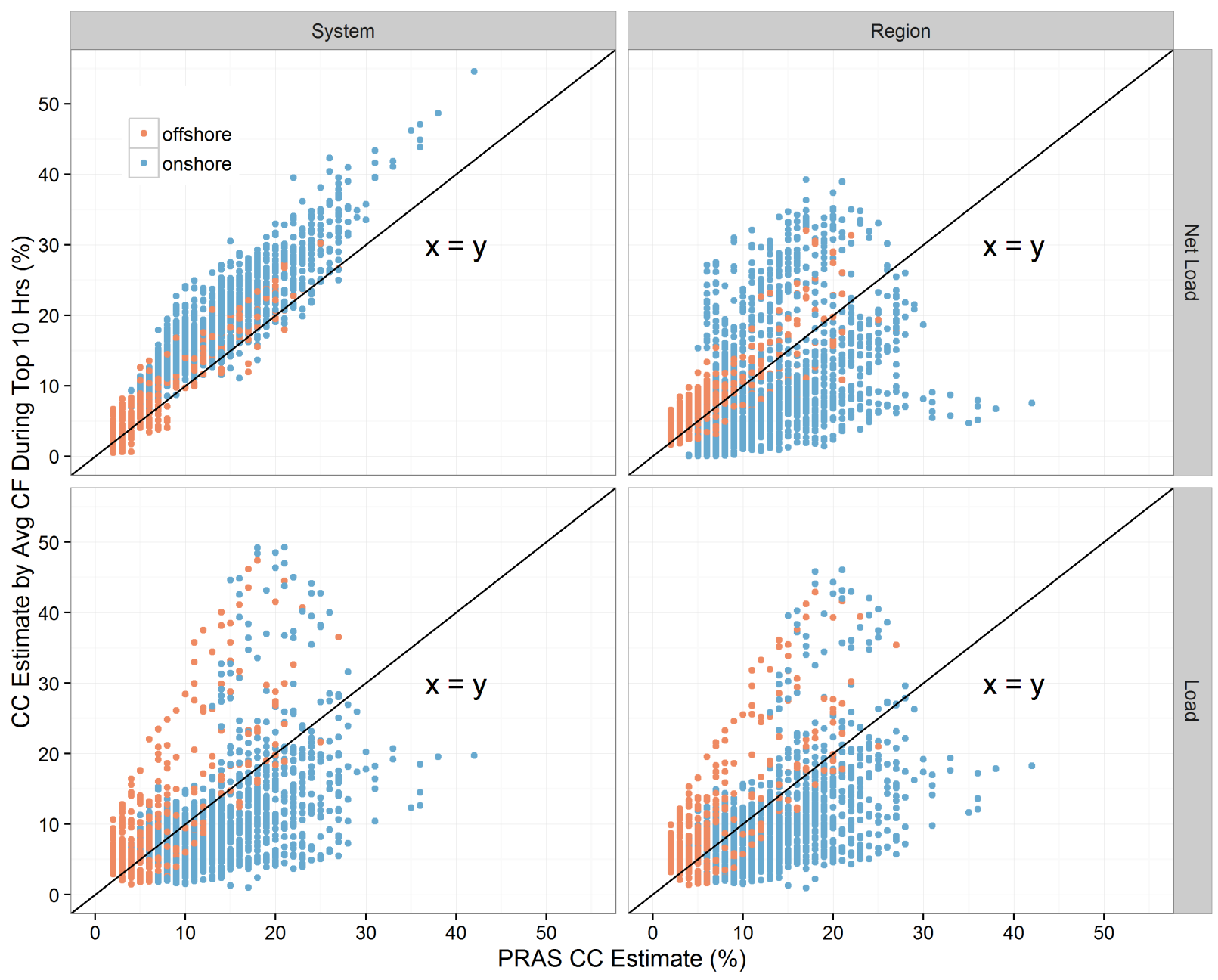

Figure 10. Comparison of marginal CC approximated by average CF during top 10 hours per year to PRAS-computed CCs.

Figure 11 illustrates a comparison of the CC computed by averaging the top system net load hours compared to the PRAS-computed CC. The bottom panels illustrate the estimated CC by averaging the CF during the top 1, 10, 100, and 1000 hours for each of the 7 years. The top panels illustrate the estimated CC by averaging the CF during the top 7, 70, 700, and 7000 hours across all 7 years together. First, comparing the upper and lower panels in Figure 11 shows that more hours (100 and 1000) makes the scatter plots similar-indicating that using a large sample (compared to averaging seven smaller ones) is less important when more hours are considered. On the other hand, having very few hours (one per year or 7 per 7 years) does seem to make a substantial difference.

The bottom four panels illustrate the estimated CC by averaging the wind CF during either the top 1 net load hour (averaging 22\%), top 10 net load hours (averaging 12.4\%), top 100 net load hours (averaging 12.7\%), and top 1000 net load in every year (averaging 14.1\%). Overall, there is not much difference between 10 and 100 hours, but there is more significant difference with 1 hour and 1000 hours. Choosing very few hours (such as 1 hour per year), seems to give an inaccurate picture of $\mathrm{CC}$, overestimating the $\mathrm{CC}$, illustrating the danger in relying too much on specific hours that might not be representative. Considering a large fraction of hours (1000, in this case) also seems to overestimate the CC by including hours that increase the average output. So, a more reasonable approach is to consider 10 or 100 hours for this system. 


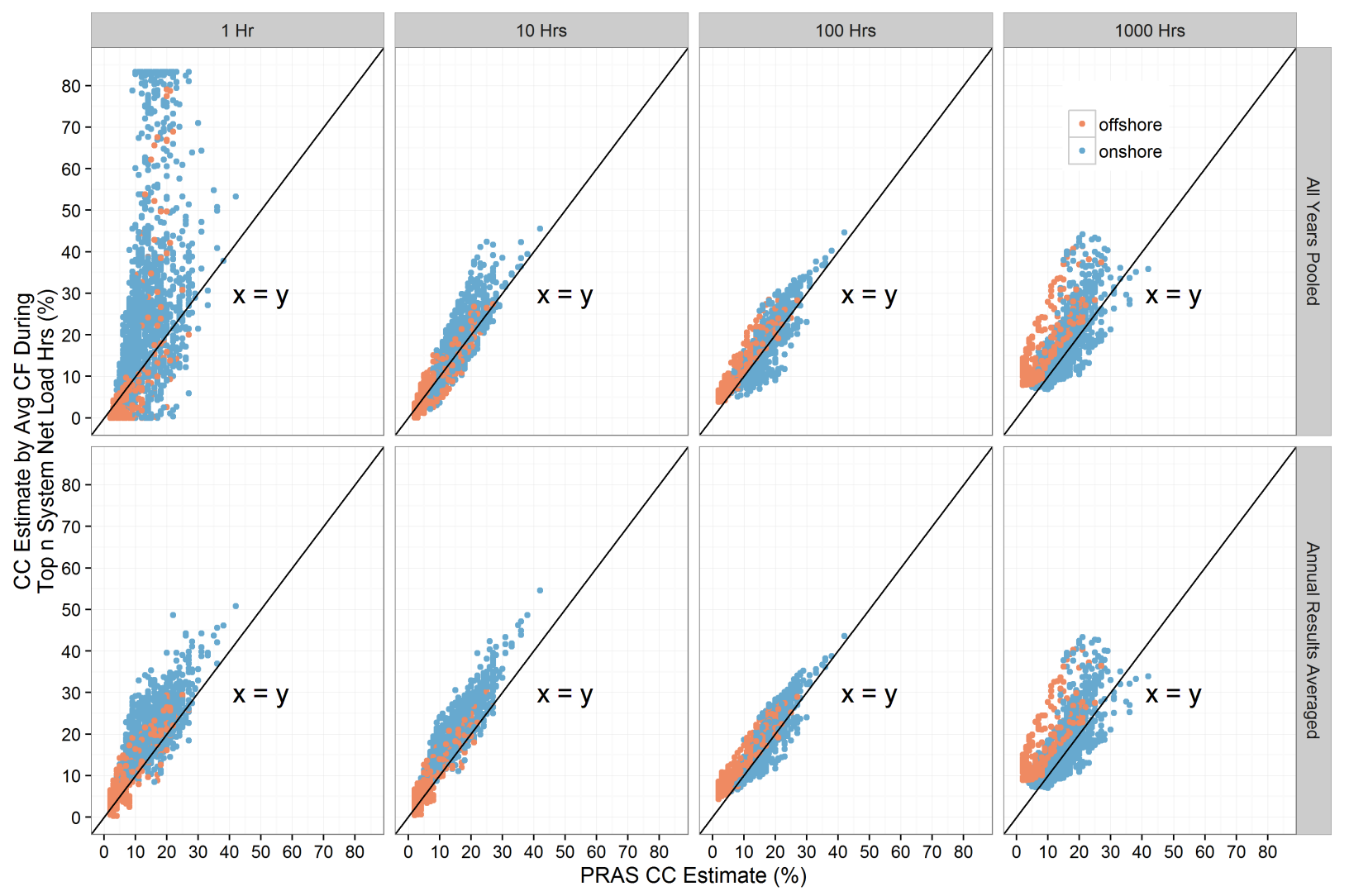

Figure 11. Comparison of CC approximated by average CF during top hours to PRAS-computed CCs.

\subsection{Computed on a Seasonal Basis}

As discussed previously, the highest EUE periods in the Texas Interconnection test system occur mostly in the summer months because of the cooling load. However, electricity demand is constantly evolving with new technology, and increased deployment of technologies such as electric heating could increase demand in (and perhaps even shift peak load into) the winter (Mai et al. 2018). Unusual cold snaps in the winter, particularly in regions with electrified heating sources, can create abnormally high demand for electricity as well. Additionally, the availability of generation resources (such as wind and solar, but also including conventional generators) can vary by season as well (Murphy, Lavin, and Apt 2020, Murphy, Sowell, and Apt 2020). These factors have led to a push to evaluate the resource adequacy implications of shifting the peak net loads to seasons other than summer (Cole et al. 2020). In fact, some organizations within the United States have started to incorporate temporal aspects into resource adequacy planning, considering needs on a monthly or seasonal basis because of varying load profiles and resource availability (PJM 2018, Operator 2020).

For the Base Case from PRAS, nearly all (99\%) observed EUE falls in the summer months (June, July, and August). Approximately $0.7 \%$ occur in the fall (September, October, and November), with the remaining $0.3 \%$ in the winter months (December, January, and February). There is zero observed EUE in the spring months (March, April, and May). We can approximate a seasonal CC by taking the system EUE-weighted CF for each of the four seasons individually. Note that this method cannot give an estimate for spring because there was zero EUE during those months. Figure 12 shows the resulting CCs. 


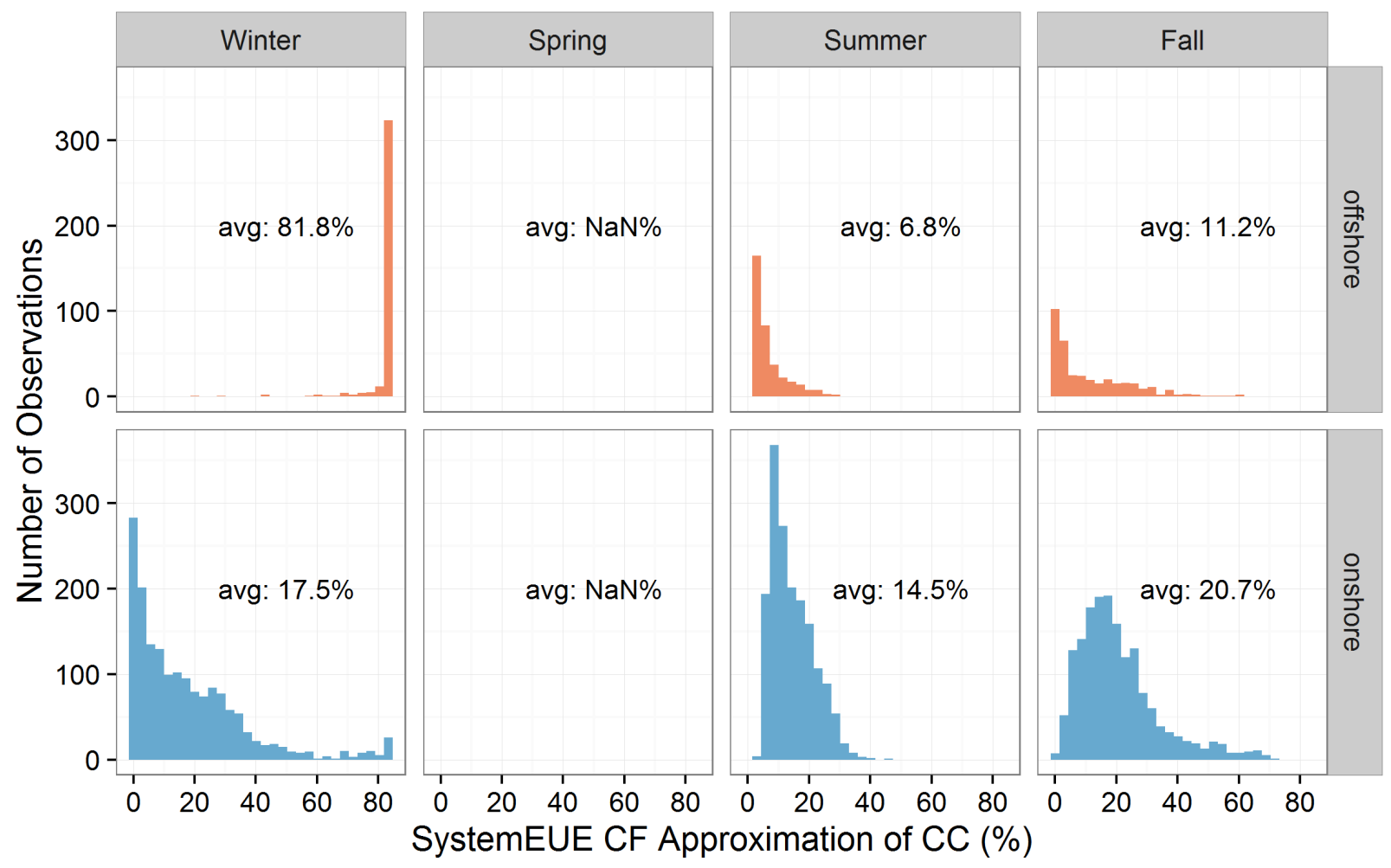

Figure 12. Comparison of CC approximated by weighting of CF by hourly Texas system-wide EUE-weighted during each of four seasons.

Both land-based and offshore wind have the lowest estimated CC in the summer. However, because summer has the most seasonal EUE, those hours are weighted the most toward the overall CC, which means that the overall CC is very close to the summer CC. We note a much higher CC for offshore wind technology in the winter (82\%), which is not surprising given the high and consistent $\mathrm{CF}$ of offshore wind during the winter months, as shown in Figure 4.

However, the winter and fall estimated CCs from Figure 12 are highly weighted to the CF in a very few number of hours, so we also compare to the $\mathrm{CC}$ approximated by averaging the $\mathrm{CF}$ during the top 10 net load hours for each season in each of the 7 years. Figure 13 shows the resulting CCs. Again, this method shows a relatively low CC during the summer, giving numbers that are very consistent with the PRAS-computed CC as well as the summer EUE estimates from Figure 12. We again note a very high CC for offshore wind technology in the winter. 


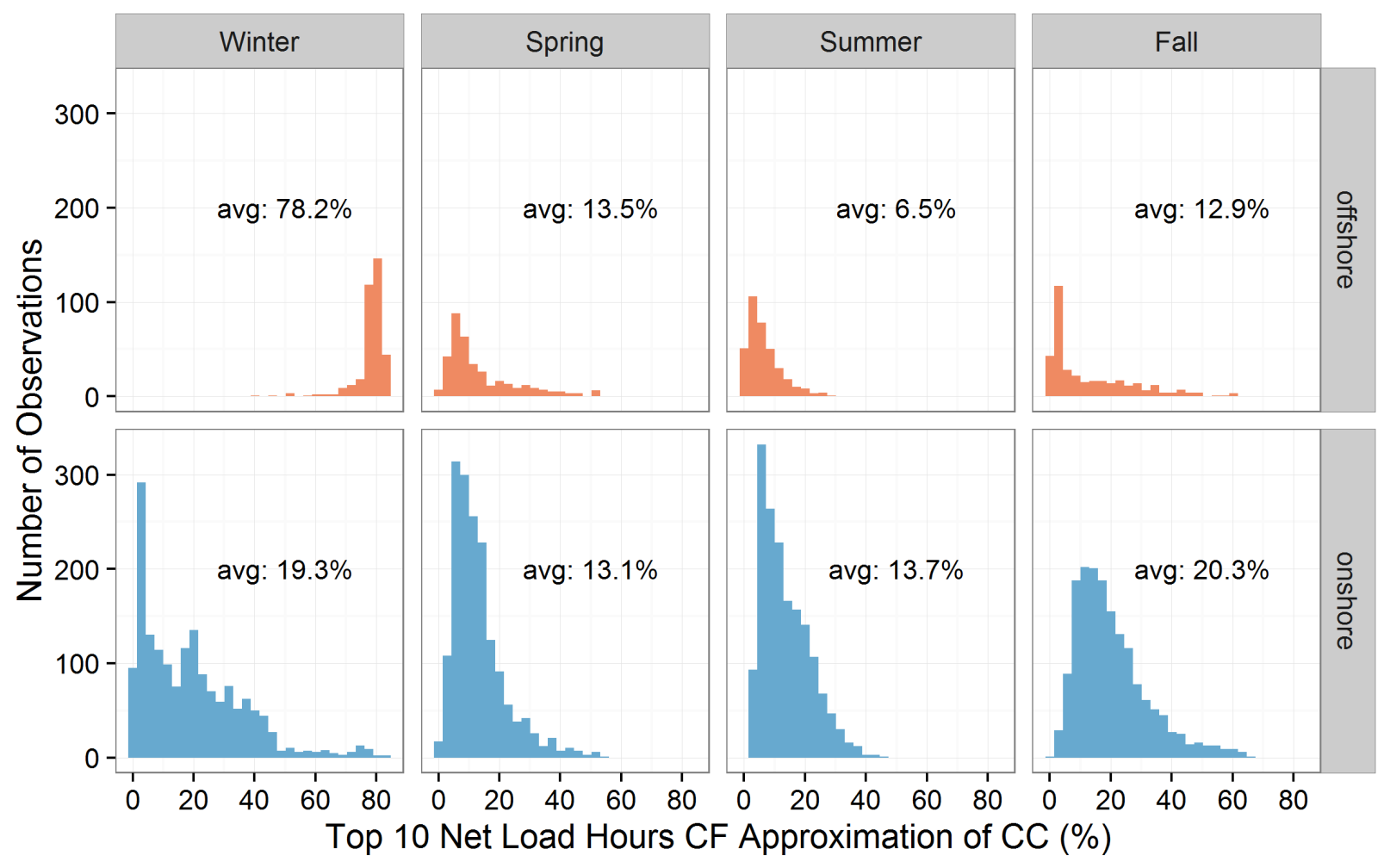

Figure 13. Comparison of CC approximated by average CF during the top 10 hours during each of four seasons.

This analysis shows dramatically different CCs for wind depending on the season considered—and these computed CCs rely solely on 7 years of historical data. Changes that are occurring in the power system could potentially shift needs to different seasons and could end up telling a different story about the marginal CC of wind in this region. Further electrification of heating load as well as extreme weather events that further increase electricity demand could underscore the importance of the seasonal consideration of resource needs in the future. 


\section{Conclusions}

Wind CC is an important metric because it describes one potential component of the value of wind, and the contribution wind provides to system resource adequacy. Our analysis examines this important metric using a Texas Interconnection test system to compare different measures of marginal wind $\mathrm{CC}$ for both land-based and offshore technologies. First, we employ a probabilistic resource adequacy tool (PRAS) to evaluate the marginal CC of landbased and offshore wind in $20-\mathrm{km}$ by $20-\mathrm{km}$ grid cells covering the footprint. We find relatively low average CCs$12.7 \%$ for land-based and $6.7 \%$ for offshore - because of a low correlation between hourly wind CF and likely times of system need. We compare this initial Base Case to various versions of the PRAS computation, varying starting generator capacity and load scaling factors to determine the sensitivity of resulting CCs. Generally, resulting wind CCs vary little between the various modeling assumptions; however, that might not be the case for all systems.

Although probabilistic resource adequacy methods can give good approximations for marginal CCs, they require relatively sophisticated tools and data sets. So, we also compare the PRAS-computed CCs with various approximation methods. Generally, we find that approximation methods can capture the important relationships between wind availability and system needs; however, the chosen approximation method can impact the resulting CCs. An important consideration is transmission - whether or not wind will be able to address the needs in neighboring regions.

Other CF approximations give reasonably similar results (especially those considering 10-100 hours per year), and consideration of seasonal or monthly CC is important because they can yield very different outcomes, especially for offshore wind. Overall, PRAS-calculated CCs seem less sensitive to modeling criteria, whereas assumptions around the CF approximation methods (such as comparing system versus regional, load versus net load) can result in varied estimations.

This analysis considers current wind generation levels in the Texas Interconnection test system (with no current offshore wind deployment) with the intent to inform near-term planning decisions and investments. We illustrate a need to continue to explore how changes in the timing of system stress (such as higher demand in winter from possible increased deployment of electric heating and extreme weather events) might change the resource adequacy role of wind in the future. Further, we will continue to explore the importance of transmission in assessing wind CC because this analysis has shown it to be an essential consideration. 


\section{References}

Awara, S. 2019. “On Capacity Value of Energy-Limited Resources in Capacity Markets”. MA thesis, Schulich School of Engineering.

Awara, S., A. J. Ardakani, H. Zareipour, and A. Knight. 2018. "Assessing Capacity Value of Wind in Alberta". AWS Truepower, L. 2015. SIMULATION OF WIND GENERATION PATTERNS FOR THE ERCOT SERVICE $A R E A$. Tech. rep. Prepared for the Electric Reliability Council of Texas (ERCOT).

Barbose, G. 2021. U.S. Renewables Portfolio Standards 2021 Annual Status Update: Early Release. Tech. rep. Lawrence Berkeley National Laboratory, Berkeley, CA (United States).

Beiter, P. C., J. K. Lau, J. E. Novacheck, Q. Yu, G. W. Stephen, J. L. Jorgenson, W. D. Musial, and E. J. Lantz. 2020. The Potential Impact of Offshore Wind Energy on a Future Power System in the US Northeast. Tech. rep. National Renewable Energy Lab.(NREL), Golden, CO (United States).

Blair, N., N. DiOrio, J. Freeman, P. Gilman, S. Janzou, T. Neises, and M. Wagner. 2018. System Advisor Model, SAM General Description (Version 2017.9.5). Tech. rep. National Renewable Energy Lab.(NREL), Golden, CO (United States).

Brinkman, G., et al. 2021. The Canadian Perspective on the North American Renewable Integration Study (NARIS). Tech. rep. National Renewable Energy Lab.(NREL), Golden, CO (United States).

Brown, M., W. Cole, K. Eurek, J. Becker, D. Bielen, I. Chernyakhovskiy, and e. a. Cohen Stuart. 2020. Regional Energy Deployment System (ReEDS) Model Documentation: Version 2019. Tech. rep. National Renewable Energy Lab.(NREL), Golden, CO (United States).

Castro, R., and L. Ferreira. 2001. "A Comparison Between Chronological and Probabilistic Methods to Estimate Wind Power Capacity Credit”. IEEE Transactions on Power Systems 16 (4): 904-909.

Cole, W., D. Greer, J. Ho, and R. Margolis. 2020. "Considerations for maintaining resource adequacy of electricity systems with high penetrations of PV and storage". Applied Energy 279:1-12.

Corporation, N. A. E. R. 2018. Probabilistic Adequacy and Measures Report. Tech. rep. NERC.

Dent, C., A. Keane, and J. Bialek. 2010. "Simplified Methods for Renewable Generation Capacity Credit Calculation: A Critical Review". In 2010 IEEE Power and Energy Society General Meeting, 1-6. IEEE.

Draxl, C., A. Clifton, B.-M. Hodge, and J. McCaa. 2015. "The Wind Integration National Dataset (WIND) Toolkit". Applied Energy 151 (1): 355-366.

Dvorak, M., B. Corcoran, J. Ten Hoeve, N. McIntyre, and M. Jacobson. 2013. "US East Coast offshore wind energy resources and their relationship to peak-time electricity demand". Wind Energy 16:977-997.

ERCOT Quick Facts. February 2021. Tech. rep. ERCOT - The Electric Reliability Council of Texas. http://www. ercot.com/content/wcm/lists/219736/ERCOT_Fact_Sheet_2.12.21.pdf.

Frew, B., G. Stephen, D. Sigler, J. Lau, W. B. Jones, and A. Bloom. 2019. "Evaluating resource adequacy impacts on energy market prices across wind and solar penetration levels". The Electricity Journal 32 (8): 106629.

Garrido, P. R. 2019. Effective Load Carrying Capability (ELCC) Review. Tech. rep. PJM.

Heath, B., and A. L. Figueroa-Acevedo. 2018. "Potential Capacity Contribution of Renewables at Higher Penetration Levels on MISO System". In 2018 IEEE International Conference on Probabilistic Methods Applied to Power Systems (PMAPS). IEEE.

Ibanez, E., and M. Milligan. 2014. Comparing resource adequacy metrics and Their Influence on Capacity Value. Tech. rep. National Renewable Energy Lab.(NREL), Golden, CO (United States).

Jorgenson, J., S. Awara, G. Stephen, and T. Mai. 2021. "A systematic evaluation of wind's capacity credit in the Western United States". Wind Energy: 1-15.

Keane, A., M. Milligan, C. J. Dent, B. Hasche, C. D’Annunzio, K. Dragoon, H. Holttinen, N. Samaan, L. Soder, and M. O’Malley. 2010. "Capacity value of wind power”. IEEE Transactions on Power Systems 26 (2): $564-572$.

Maclaurin, G., N. Grue, A. Lopez, and D. Heimiller. 2019. The Renewable Energy Potential (reV) Model: A Geospatial Platform for Technical Potential and Supply Curve Modeling. Tech. rep. National Renewable Energy Lab.(NREL), Golden, CO (United States).

Madaeni, S., R. Sioshansi, and P. Denholm. 2012. "Estimating the Capacity Value of Concentrating Solar Power Plants: A Case Study of the Southwestern United States". IEEE Transactions on Power Systems 27 (2): 1116 1124. 
- . 2013. "Comparing Capacity Value Estimation Techniques for Photovoltaic Solar Power". IEEE Journal of Photovoltaics 3 (1): 407-415.

Mai, T., P. Jadun, J. Logan, C. McMillan, M. Muratori, D. Steinberg, L. Vimmerstedt, R. Jones, B. Haley, and B. Nelson. 2018. Electrification Futures Study: Scenarios of Electric Technology Adoption and Power Consumption for the United States. Tech. rep. National Renewable Energy Lab.(NREL), Golden, CO (United States).

Milligan, M. 1997. A comparison and case study of capacity credit algorithms for intermittent generators. Tech. rep. National Renewable Energy Lab., Golden, CO (United States).

- . 2000. "Modelling Utility-Scale Wind Power Plants. Part 2: Capacity Credit". Wind Energy 3:167-206.

Milligan, M., B. Frew, E. Ibanez, J. Kiviluoma, H. Holttinen, and L. Söder. 2017. "Capacity value assessments of wind power". Wiley Interdisciplinary Reviews: Energy and Environment 6 (1): e226.

Milligan, M., and K. Porter. 2008. Determining the capacity value of wind: An updated survey of methods and implementation. Tech. rep. National Renewable Energy Lab.(NREL), Golden, CO (United States).

Mills, A., and P. Rodriguez. 2019. Drivers of the Resource Adequacy Contribution of Solar and Storage for Florida Municipal Utilities. Tech. rep. Lawrence Berkeley National Laboratory(LBNL), Berkeley, CA (United States).

Mills, A., and R. Wiser. 2012. Changes in the Economic Value of Variable Generation at High Penetration Levels: A Pilot Case Study of California. Tech. rep. Ernest Orlando Lawrence Berkeley National Lab (LBNL), Berkeley, CA (United States).

Mills, A. D., and P. Rodriguez. 2020. "A simple and fast algorithm for estimating the capacity credit of solar and storage". Energy 210:118587.

Murphy, S., L. Lavin, and J. Apt. 2020. "A time-dependent model of generator failures and recoveries captures correlated events and quantifies temperature dependence". Applied Energy 262:1-14.

Murphy, S., F. Sowell, and J. Apt. 2020. "Resource adequacy implications of temperature-dependent electric generator availability". Applied Energy 253:1-11.

Musial, W., P. Beiter, J. Stefak, G. Scott, D. Heimiller, T. Stehly, S. Tegen, O. Roberts, T. Greco, and D. Keyser. 2020. Offshore Wind in the US Gulf of Mexico: Regional Economic Modeling and Site-Specific Analyses. Tech. rep. National Renewable Energy Lab.(NREL), Golden, CO (United States).

New England Wind Integration Study. 2010. Tech. rep. GE Energy.

Operator, C. I. S. 2020. Final Flexible Capacity Needs Assessment for 2021. Tech. rep. http://www. caiso .com/ Documents/Final2021FlexibleCapacityNeedsAssessment.pdf.

PJM. 2018. Intermittent Resource Participation in RPM for 2020/21 and beyond. Tech. rep. https://www.pjm.com//media/committees-groups/subcommittees/irs/20180305/20180305-item-10-intermittent-resource-participationin-rpm.ashx.

Sengupta, M., Y. Xie, A. Lopez, A. Habte, G. Maclaurin, and J. Shelby. 2018. "The National Solar Radiation Database (NSRDB)". Renewable and Sustainable Energy Reviews 89:51-60.

Sioshansi, R., S. H. Madaeni, and P. Denholm. 2013. "A dynamic programming approach to estimate the capacity value of energy storage". IEEE Transactions on Power Systems 29 (1): 395-403.

Soder, L., and M. Amelin. 2008. "A review of different methodologies used for calculation of wind power capacity credit". In 2008 IEEE Power and Energy Society General Meeting-Conversion and Delivery of Electrical Energy in the 21st Century, 1-5. IEEE.

Stehly, T., P. Beiter, and P. Duffy. 2020. 2019 Cost of Wind Energy Review. Tech. rep. National Renewable Energy Lab.(NREL), Golden, CO (United States).

Stephen, G. 2019. Probabilistic Resource Adequacy Suite (PRAS). USDOE Office of Renewable Energy and Energy Efficiency. doi:10.11578/dc.20190814.1. https://www.osti.gov//servlets/purl/1557438.

- . 2021. Probabilistic Resource Adequacy Suite (PRAS) v0.6 Model Documentation. Tech. rep. National Renewable Energy Lab.(NREL), Golden, CO (United States).

Stephen, G., E. Hale, and B. Cowiestoll. 2020. Managing Solar Photovoltaic Integration in the Western United States: Resource Adequacy Considerations. Tech. rep. National Renewable Energy Lab.(NREL), Golden, CO (United States).

The Electric Reliability Council of Texas. 2021. "February 2021 Extreme Weather Event". http://www.ercot.com/ news/february2021\%.

U.S. Energy Information Administration. 2021. "ELECTRICITY Form EIA-860 detailed data with previous form data (EIA-860A/860B)". https://www.eia.gov/electricity/data/eia860/. 